\title{
Frequency and phase locking of laser cavity solitons
}

\author{
T. Ackemann, Y. Noblet, P. V. Paulau, C. McIntyre, P. Colet, W. J. Firth, G.-L. \\ Oppo
}

\begin{abstract}
Self-localized states or dissipative solitons have the freedom of translation in systems with a homogeneous background. When compared to cavity solitons in coherently driven nonlinear optical systems, laser cavity solitons have the additional freedom of the optical phase. We explore the consequences of this additional Goldstone mode and analyze experimentally and numerically frequency and phase locking of laser cavity solitons in a vertical-cavity surface-emitting laser with frequency-selective feedback. Due to growth-related variations of the cavity resonance, the translational symmetry is usually broken in real devices. Pinning to different defects means that separate laser cavity solitons have different frequencies and are mutually incoherent. If two solitons are close to each other, however, their interaction leads to synchronization due to phase and frequency locking with strong similarities to the Adler-scenario of coupled oscillators.
\end{abstract}

\section{Introduction}

Lasers are a prominent example for self-sustained nonlinear oscillators. The phase of the oscillation of the electromagnetic field is not determined but results from the spontaneous breaking of the time symmetry present for $\mathrm{cw}$ pumping. Hence, the

T. Ackemann, Y. Noblet, C. McIntyre, W. J. Firth, G.-L. Oppo,

SUPA and Department of Physics, University of Strathclyde, Glasgow G4 0NG, Scotland, UK. email: thorsten.ackemann@strath.ac.uk, yoann.noblet@strath.ac.uk, craig.mcintyre@ strath.ac.uk, willie@phys.strath.ac.uk, g.l.oppo@strath.ac.uk

P. V. Paulau

TU Berlin, Institut für Theoretische Physik, Hardenbergstr. 36, Sekr EW 7-1, 10623 Berlin, Deutschland. e-mail: pavel.paulau@tu-berlin.de

P. Colet

IFISC, (CSIC-UIB), Campus Universitat Illes Balears, E-07071 Palma de Mallorca, Spain. e-mail: pere@ifisc.uib-csic.es 
phase is a Goldstone mode of the dynamics and couples easily to perturbations, such as, for example, spontaneous emission. As a result of the induced 'phase diffusion' [1], a laser has a finite linewidth (Schalow-Townes limit) and the mutual coherence between two independent lasers will be limited even if they have the same center frequency. Hence a lot of attention has been given to the phenomena of frequency and phase locking, by which coupled lasers can synchronize their frequency and phases to achieve coherent emission [2, 3, 4,5]. Laser synchronization is just a special example of synchronization dynamics of coupled oscillators, which is of high importance in all fields of Nonlinear Science [6, 7], the earliest example being the famous observation made by Christiaan Huygens on two pendulum clocks. A very well known biological example involving a large number of oscillators is the synchronized flashing of fireflies [6,7].

In photonics, frequency and phase locking has particular relevance for laser injection $[8,9]$ and in laser arrays where it can establish coherence between individual emitters even in the presence of disorder $[10,11,12]$, i.e. an unintentional variation of the frequencies of the free-running emitters forming the array. Phase-locking of disorder-induced localized modes was observed in microchip lasers [13]. Synchronization of chaotic lasers has promises for secure communication [14, 15, 16, 17].

One other object of fundamental importance in different areas of Nonlinear Science is the soliton $[18,19,20]$, i.e. a nonlinearly self-localized state in one or more dimensions in a conservative or dissipative system. As a self-localized state it can exist anywhere in a translationally invariant system. Hence, laser solitons [21, 22] have the freedom of translation in an ideally homogeneous system typical of solitary waves as well as the freedom to choose the oscillation phase typical of lasers. Both are Goldstone modes of the dynamics. Their interplay involves aspects of synchronization dynamics and of soliton interaction. Dissipative solitons in coherently driven optical systems [23] (Fig. 1a)- i.e. without the phase degree of freedom display a peculiar interaction behavior with a set of bound states with different, discrete distances between constituents [24, 25, 26, 27], which is also typical of many non-optical systems [28], and is related to modulated tails of the solitons. Propagation solitons in conservative system like the Nonlinear Schrödinger equation show phase-sensitive interaction behavior (attraction for zero relative phase, repulsion for $\pi$ relative phase) [18]. In the present case of a soliton laser, phase and location are both dynamical variables.

As a consequence of synchronization and interaction, phase-locked bound states with well defined phases and distances of the constituent laser solitons have been predicted to form in simple model systems like the cubic-quintic Ginzburg Landau equation (CQGLE) [29, 30, 31, 32, 33, 34, 35, 36]. These works concentrate on one-dimensional dissipative solitons being motivated by temporal solitons in modelocked lasers (Fig. 1d). Indeed corresponding bound states were observed experimentally in fiber lasers [37, 26, 38, 39]. A recent review of these phenomena is in [40].

Until recently the spatial case had been addressed only theoretically in lasers with saturable absorbers (LSA) (Fig. 1c) [41, 21, 42]. Early experiments on solitons in LSA using dyes and photorefractives as gain medium were limited to one soliton due 


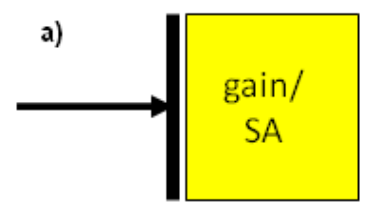

b)

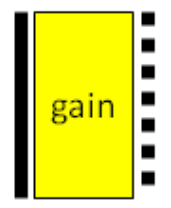

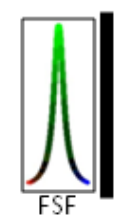

c)

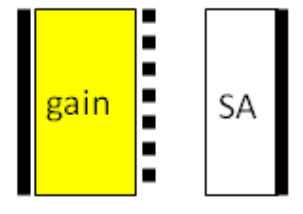

d)

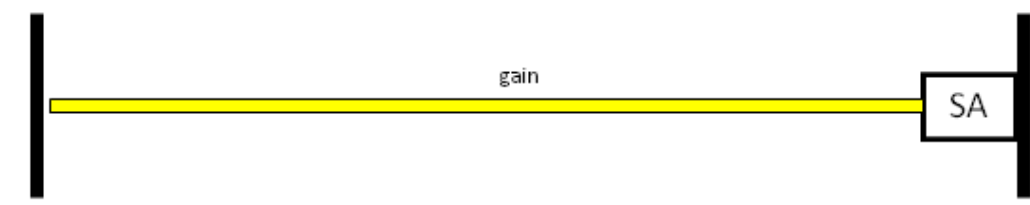

Fig. 1 Optical schemes displaying dissipative solitons. Panels a-c illustrate different schemes for spatial solitons where the gain cavity is short to achieve a high Fresnel number or aspect ratio (ideally single longitudinal mode, for simplicity and the validity of a uniform field approximation), whereas scheme d illustrates a scheme for temporal solitons as, e.g., a fiber laser, where the cavity is long and highly multi-longitudinal mode. $\mathrm{SA}=$ saturable absorber, $\mathrm{FSF}=$ frequency selective feedback, thick black lines: cavity mirrors, dashed lines: partial reflectors (in scheme c the partial reflector is not mandatory but present in many experimental realizations). In panel a, illustrating an amplifier (or a driven passive cavity), the phase symmetry is broken due to an external coherent field.

to specific cavity geometries [43, 44, 45]. Later investigations in a photorefractive oscillator found multiple spatial laser solitons but did not investigate the interaction and phase properties [46]. Recently, there has been strong progress in the realization of spatial laser solitons in semiconductor-based micro-resonators with frequencyselective feedback $[47,48,49,50,51,52]$ and saturable absorption $[53,54,55$, $56,42]$. In these systems, a broad transverse area (transverse indicates the plane orthogonal to the cavity axis) is pumped in a marginally stable plano-planar cavity. Emission does not take place over the whole aperture but on filaments which are much smaller than the pumped aperture. These filaments are dissipative solitons. We will refer to them as laser cavity solitons (LCS) and to the device as a cavity soliton laser (CSL). Recent reviews are in [57, 58].

LCS represent small coherent emitters, i.e. microlasers [47, 49]. Spatially separated LCS are usually found to be incoherent in experiments $[49,59]$. This is due to uncontrolled fluctuations in the expitaxial growth process, which cause a variation of resonance conditions across the pumped aperture of the device. Since translation is a Goldstone mode of a soliton, it will couple to all spatially inhomogeneous perturbations and the soliton will move until it reaches a local extremum of the perturbation, where the gradient vanishes [60,61]. This leads first to a pinning of the solitons at certain positions generally referred as either traps or defects. This was investigated in detail in coherently driven semiconductor microcavities [62, 63] (Fig. 1a). More recently, frequency-selective feedback (Fig. 1b) to a laser device was shown to provide simple means for mapping these variations [64].

Background defects in lasers not only fix LCS position but also induce a shift in the LCS natural frequency. Recently it was shown that this diversity in natural 
frequencies among LCS pinned by defects is a critical ingredient in the description of their interaction and synchronization in real systems, leading to a scenario quite different from the CQGLE on a homogenous background [65]. Ref. [65] - which we review and extend here - presents experimental and theoretical evidence that the interaction of spatial LCS in real lasers is governed by the archetypal Adler locking mechanism [66]. The Adler locking mechanism has relevance in biological clocks, chemical reactions, mechanical and electrical oscillators [6]. In optics frequency locking of the Adler type was first observed in lasers with injected signals [9] with more recent generalizations to coupled lasers [4], the spatio-temporal domain [67], quantum dot lasers [68] and frequency without phase locking [69].

We note that for temporal LCS, such as those arising in fiber lasers [37, 26, 38] and driven fiber cavities [70,71], the effects of longitudinal inhomogeneities are washed out by the propagation dynamics along the cavity axis (see Fig. 1d). Thus every soliton sees the same material characteristics [72]. Hence, theoretical studies considering the interaction of identical LCS arising on a homogeneous background seem to be suitable for temporal LCS, but are not adequate to describe the dynamics of coupled spatial LCS. Temporal solitons would be affected by copropagating inhomogeneities [73, 74] which can be induced by modulating laser parameters $[75,76,77,78]$. These regular parameter modulations were shown to have a substantial effect on the phase-locked bound states but do not induce frequency detunings between solitons when these are assembling at the minima of the potential. Hence the relevance of frequency and phase locking and the Adler scenario was not addressed in these works.

The organization of this book chapter is as follow. In the next section, Sect. 2, we introduce the experimental system, a vertical-cavity surface-emitting laser (VCSEL) coupled to a volume Bragg grating (VBG) as a frequency-selective element (Fig. 1b). We review basic features and observations in this system and then provide evidence and a detailed analysis of frequency and phase locking. From a dynamical point of view, VCSELS can be considered as Class B lasers characterized by the fact that the dynamics can be described in terms of the complex optical field and population inversion while the material dielectric polarization can be adiabatically eliminated. For a theoretical and numerical description of the system, we discuss in Sect. 3 a class B-laser model [52]. This model is simplified afterwards to a complex equation for the optical field alone, which is a Ginzburg-Landau equation with an additional linear filter (GLE-F) $[79,80]$ and provides the simplest framework to understand the observed dynamics. In Sect. 4 we study first the interaction of LCS on a homogeneous background in the GLE-F and find a close correspondence to the results predicted by the CQGLE. We will see that some of the LCS interaction properties on a homogeneous background are significantly modified in the classB model that works for more realistic parameters. Then, in Sect. 5, we investigate the case of LCS interaction in the presence of inhomogeneities, describe the resulting Adler-synchronization for both models and compare the results to experiments. Sect. 6 provides then a final discussion and outlook. 


\section{Laser cavity solitons and their interactions in VCSELs with feedback}

\subsection{Devices and experimental setup}

A vertical-cavity surface-emitting laser (VCSEL) is a semiconductor laser in which the emission is in the direction of the epitaxial growth (see Fig. 2a). The VCSEL used for this experiment is similar to the ones described in more detail in $[81,82,49,83]$. Three InGaAs quantum wells are serving as gain medium leading to emission in the $980 \mathrm{~nm}$ range. The quantum wells are surrounded by passive AlGaAs spacer layers with a total thickness of one wavelength. The cavity is closed by high reflectivity distributed Bragg reflectors (DBR) with 33 layers AlGaAs/GaAs on the top side (p-contact) and 22 layers on the bottom side (n-contact). The emission takes place through the $n$-doped Bragg reflector and through the transparent substrate. In this so-called bottom-emitting geometry a reasonable uniformity of carrier injection can be achieved over fairly large apertures [81, 82]. A $200 \mu \mathrm{m}$ diameter circular oxide aperture provides optical and current guiding. This active diameter is much larger than the effective cavity length of about $1.2 \mu \mathrm{m}$. As a result, the VCSEL has a large Fresnel number allowing for the formation of many transverse cavity modes of fairly high order.

a)

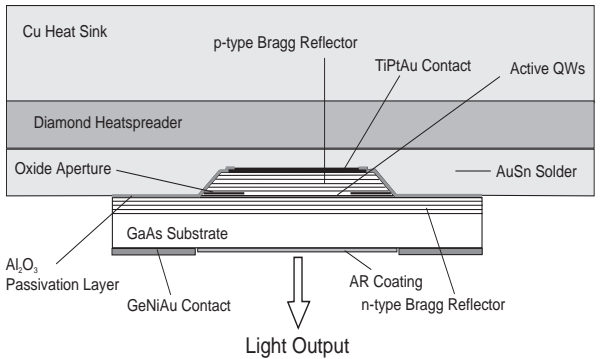

b)

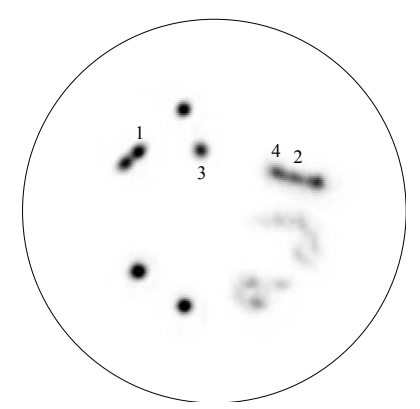

Fig. 2 a) Schematic diagram of VCSEL layer structure. [From [84]]. b) Near field image of the VCSEL aperture taken at $396 \mathrm{~mA}$ showing the relative position and numbering of a few solitons. Output VCSEL images, like all in the following, are in a linear gray scale with black denoting high intensity.

The laser has an emission wavelength around $975 \mathrm{~nm}$ at room temperature. Frequency-selective feedback is provided by an external volume Bragg grating (VBG). The VBG has a reflection peak at $\lambda_{\mathrm{g}}=981.1 \mathrm{~nm}$ with a reflection bandwidth of $0.2 \mathrm{~nm}$ full-width half-maximum (FWHM). The VCSEL is tuned in temperature up to $70^{\circ} \mathrm{C}$ so that the emission wavelength approaches the reflection peak of the VBG. At such a high temperature the free running laser has an infinite threshold and lasing only occurs because of the feedback from the VBG. 
A schematic diagram of the experimental setup is illustrated in Fig. 3. The VCSEL is coupled to the VBG via a self-imaging external cavity. Every point of the VCSEL is imaged at the same spatial position after each round trip therefore maintaining the high Fresnel number of the VCSEL cavity and ensuring local feedback compatible with self-localization.

The VCSEL is collimated by $f_{1}=8 \mathrm{~mm}$ focal length plano-convex aspheric lens. The second lens is a $f_{2}=50 \mathrm{~mm}$ focal length plano-convex lens and is used to focus the light onto the VBG. This telescope setup gives a $6.25: 1$ magnification factor onto the VBG. This cavity has a round trip frequency of $1.23 \mathrm{GHz}$ which corresponds to a round trip time of $0.81 \mathrm{~ns}$. The light is coupled out of the cavity using a glass plate (beam splitter with a front uncoated facet and a back anti-reflection coated facet). The reflection is relying on Fresnel reflection and therefore is polarization dependent. The reflectivity is on the order of $10 \%$ for s-polarized light and $1 \%$ for p-polarized light.

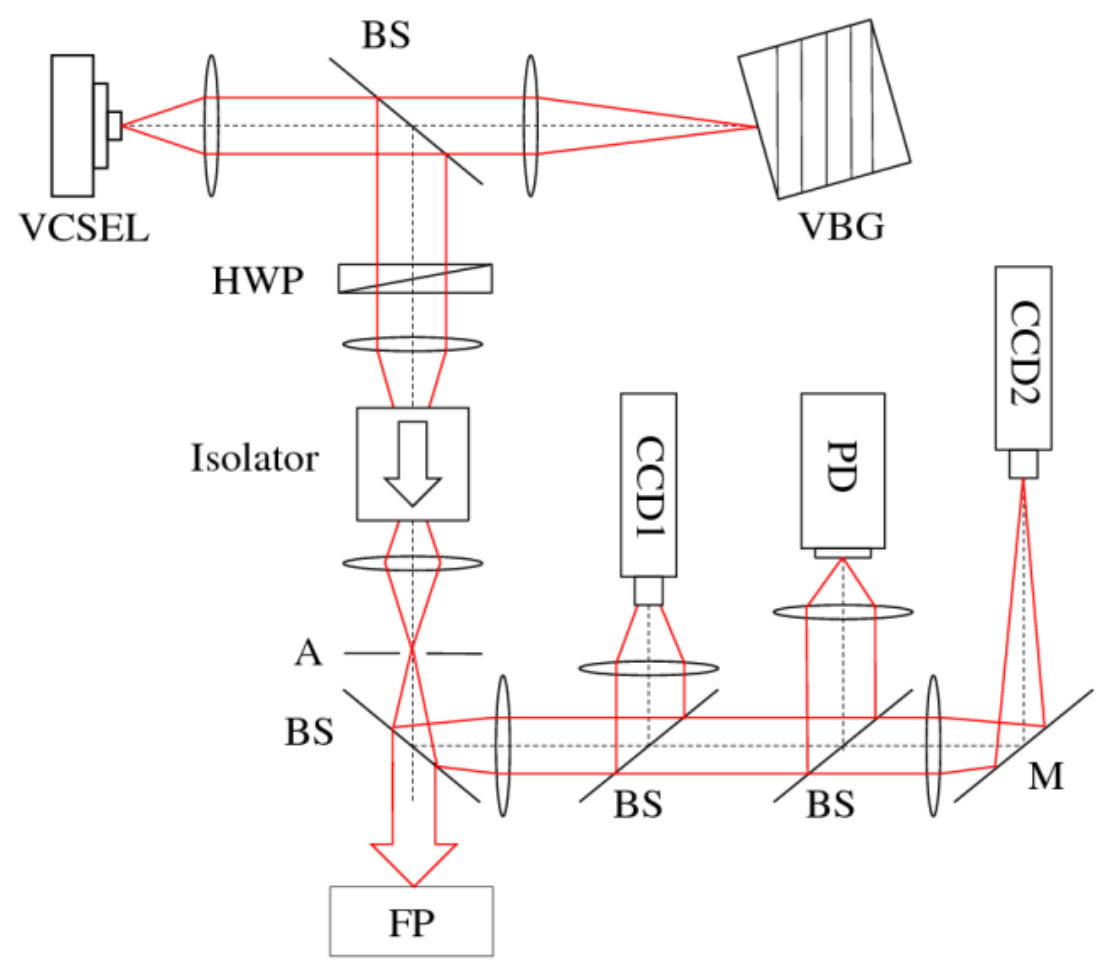

Fig. 3 Experimental setup. VCSEL: Vertical-cavity surface-emitting laser, BS: Beam splitter, VBG: Volume Bragg grating, HWP: Half wave plate, A: Aperture, M: Mirror, PD: Photo diode, CCD1: CCD camera in near field image plane of VCSEL, CCD2: CCD camera in far field image plane of VCSEL, FP: Fabry-Perot. 
An optical isolator is used to prevent reflection from the detection system to pass into the external cavity. There are two charge-coupled-device (CCD) cameras used for detection, one is used to produce images of the VCSEL emission in the gain region (near field) and the other camera produces images of the Fourier plane of the gain region (far field). The optical spectrum is recorded with a scanning FabryPerot interferometer (FP). It has free spectral range (FSR) of $10 \mathrm{GHz}$ and a maximal Finesse of 80 . There is also a photodiode which measures the total laser power.

Fig. 4 Scheme of VCSEL cavity carrying two LCS with feedback from a tilted VBG. The tilt angle $\beta$ of the VBG controls the mutual detuning of the two LCS (see text for details). The pivot point is much further away from the optical axis in reality (about $30 \mathrm{~mm})$.

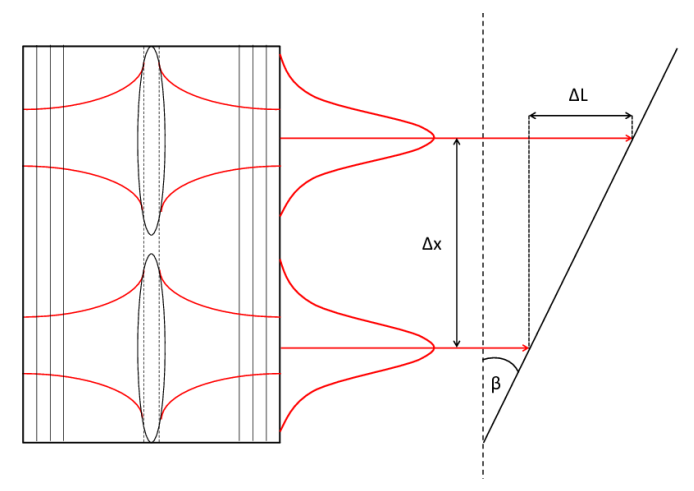

As it will be discussed in more detail in the following, the precise alignment of the VBG is very important. It is controlled by fine adjustment screws. The tilt $\beta$ (see Fig. 4) in the horizontal direction can be fine-adjusted by a piezo-electric transducer (PZT), which is stabilized against drift by a servo-loop controlled by a strain gauge. A computer-controlled voltage $U$ applied to the PZT leads to a tilt of $\delta \beta / \delta U=2.1 \times 10^{-5} / \mathrm{V}$, a change of the external cavity length at a rate of $\delta L / \delta U=0.628 \mu \mathrm{m} / \mathrm{V}$, and a change of the resonance frequency in the external cavity by $\delta v / \delta U=1.576 \mathrm{GHz} / \mathrm{V}$. Perhaps even more importantly for what follows, there is also a differential shift for two LCS. If their distance projected onto a plane orthogonal to the rotation axis is $\Delta x$, this shift is

$$
\Delta v=\Delta x \frac{2 v_{F S R} \sin \beta}{\lambda}
$$

where $v_{F S R}$ is the free spectral range of the external cavity and $\lambda$ the operating wavelength of the VCSEL. The change of the ray angle after returning to the VCSEL (corrected for the magnification of the telescope) is $\theta=\beta \mathrm{M}$.

The adjustment of the self-imaging condition is described in detail in [85]. The distance between the VCSEL and the collimating lens can be adjusted for best collimation, while the distance between the focusing lens and the VBG is selected by adjusting the images of the VCSEL emission at high current for maximum sharpness of the aperture. For the distance between the two lenses there is no simple alignment criterion since it needs to be set from the lens specifications taking the dispersion data of the lenses into account. Then it can be checked by measuring dispersion 
curves of the off-axis states above threshold [85]. After optimization we estimate to be within $\pm 0.2 \mathrm{~mm}$ of the self-imaging condition with a reasonable depth of focus [85].

\subsection{Basic properties of laser cavity solitons}

When increasing the VCSEL input current, LCS appear at specific spatial locations. The first lasing emission occurs at currents of about 360-380 mA, depending on the exact temperature and alignment of the VBG. Fig. 2b shows a typical near field intensity distribution slightly above threshold. There are several distinct spots of emission, which are approximately circularly symmetric and approximately equal in amplitude and shape. These are the LCS. The size of a LCS is about 5-7 $\mu \mathrm{m}$ $\left(1 / e^{2}\right.$-radius). The far field has also a single-lobe, well-behaved profile with a width of a few 10s of mrad [49], i.e. the LCS have a high spatial coherence. The emission is also temporally coherent with a typical linewidth of about $6 \mathrm{MHz}$ when operating on a single longitudinal mode of the external cavity [49], quite a typical value for grating-controlled lasers on millisecond and second time scales. Hence each LCS is a coherent emitter, a micro-laser. We will discuss the mutual coherence of LCS below. By increasing the current, a soliton typically splits into a compound state with two humps, and then possibly three or four, followed by disordered extended states. Evidence for these states, stemming from the LCS with the lowest threshold, is visible in the lower right part of the aperture in Fig. 2b. Details on the LCS and pattern evolution beyond threshold can be found in [49, 85].

The appearance of each LCS is abrupt and we observe hysteresis when the current is ramped up and down, i.e., each LCS shows bistability. Fig. 5 illustrates this phenomena for two sample LCS shown in the corresponding inset. The right one switches on abruptly at about $379.5 \mathrm{~mA}$, the left one only slightly later at about $381 \mathrm{~mA}$. If the current is decreased again, the latter survives till about $376 \mathrm{~mA}$, the former till $372.5 \mathrm{~mA}$. In between 376 and $397.5 \mathrm{~mA}$ the two LCS are simultaneously and individually bistable, i.e. they can be independently switched on and off by an external writing pulse [47, 48, 49]. This represent a 2-bit memory, which is interesting for optical information processing when extended to more channels that can display the potential for massive parallelism in broad-area VCSELs. The details of this hysteresis loops are different for different LCS and also depend on alignment. Typically switch-on occurs to the single-humped fundamental LCS as demonstrated in Fig. 5. Corresponding scenarios are described in [47, 48, 49, 86], but a direct transition to multi-humped and ring-shaped states is also possible for, e.g., solitons 1 and 2. The experiments described later in Sects. 2.3 are performed at a bias current at which two fundamental, single-humped LCS are individually bistable.

The mechanism for the bistability is the following [87, 88, 48, 85]. Initially, the longitudinal resonance of the cavity is blue-detuned to (at higher frequency than) the grating frequency (the reflection peak of the grating). Hence there is a frequency gap, in which no linear state exists. The gain is below the value where lasing without 
Fig. 5 Light-current (LI) curve for two sample LCS (shown in inset). The solid line refers to increasing current and the dashed line to decreasing current. Measurements taken at $0.1 \mathrm{~mA}$ intervals at a rate of $1 \mathrm{~mA}$ per second to avoid thermal hysteresis.

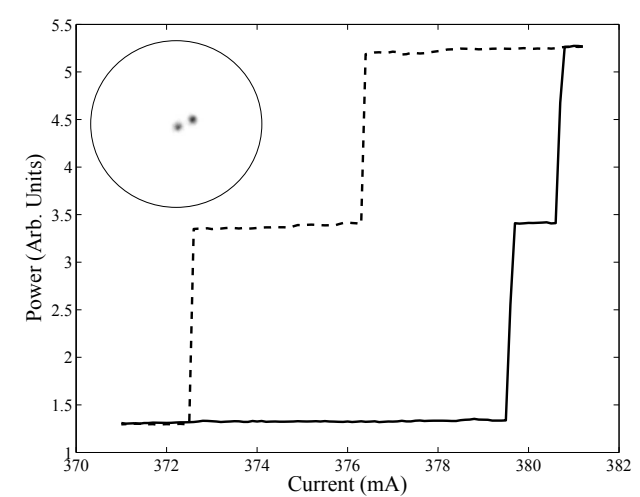

the help of the feedback from the grating is possible. Increasing the current leads to an increase of Ohmic dissipation and hence to a temperature rise in the laser structure. This results in an increase of the refractive index and hence in a red-shift of the cavity resonance. This shift is around $0.0035 \mathrm{~nm} / \mathrm{mA}$. Let's assume now a fluctuation leading to an increase in output power. Due to stimulated emission, the carrier density is decreased and this increases the refractive index due to phase-amplitude coupling in semiconductors, described phenomenologically by Henry's alpha-factor [89]. This red-shifts the cavity resonance and hence the detuning between VCSEL and VBG decreases. As a result, the feedback strength increases and the intensity will increase ever further leading to positive feedback. At a certain critical detuning, the positive feedback is strong enough to cause an abrupt switch-on of the laser. After switch-on, the laser can self-sustain the condition of the near-resonance between the (shifted) cavity resonance and the VBG in the high-amplitude emission state due to the continuous depletion of carriers and the resulting refractive index shift even if the current is reduced again, resulting in a bistable situation. Bistability due to shifts of cavity resonances is usually referred to as dispersive optical bistability [90].

In a spatially extended system, the whole aperture might switch to the highamplitude state, but it turns out that this state is unstable, or at least that there is the coexisting possibility of localized emission, the LCS [51, 52]. An intuitive mechanism to understand the drive for localization is self-lensing, as indicated in Fig. 4. A self-induced lens can render the marginally stable plano-planar resonator locally stable and lead to a self-induced nonlinear mode, the LCS [91, 25]. It is important to realize that under our typical operating conditions the main effect of the change of the control parameter 'current' (see e.g. Fig. 5) is the change of the detuning condition and not the gain change. This is further evidenced by the fact that a decrease of the ambient temperature of the VCSEL leads to an increase in threshold current. Since the gain at constant current increases in a semiconductor with decreasing temperature, this indicates that the main function of the increased current is to provide the larger detuning shift required at lower temperature. The system with feedback by a normal mirror lases already at about $180 \mathrm{~mA}$ [85], demonstrating that ample gain is available. 
The reason for the difference in threshold for the different solitons as well as for the preference of certain locations lies in growth irregularities of the semiconductor material. If the cavity resonance of the VCSEL is spatially varying, the linear gap between the grating frequency and the cavity resonance is changing in space and a minimum value of the detuning for switch-on is met at different locations or different injection currents. Hence the lasing will start first at the most 'reddish' location with the smallest gap. With increasing currents more locations reach the critical detuning value and additional LCS switch on, whereas the LCS formed originally may give way to high-order compound states and extended, off-axis lasing states $[64,85]$. Typical length scales of disorder are about $10 \mu \mathrm{m}$ and they span some tens of $\mathrm{GHz}$ [64] in line with the results from other devices in the literature [63, 92, 93]. A simple calculation shows that a monolayer variation of $\Delta L \approx 0.3 \mathrm{~nm}$ corresponds to a frequency variation of $\Delta v \approx 76 \mathrm{GHz}$. Similar results are obtained with more accurate models of the multi-layer stacks forming a VCSEL [94, 95]. Since the cavity linewidth of a low-loss VCSEL is about $0.1 \mathrm{~nm}$ or $30 \mathrm{GHz}$ (depending on the actual free-carrier and scattering losses), this explains the extreme sensitivity of the feedback light distribution to disorder.

As explained in the introduction, LCS will couple to parameter variations and drift $[60,61]$ until they either disappear from the system or reach a point in which all first order perturbations, i.e. gradients, vanish at a local extremum of the 'landscape' imposed by the variations. These preferred locations are the ones where we find LCS in Fig. 2b. We will refer to them as traps or defects. This disordered 'landscape' is frozen after the growth process of the semiconductor structure although some minor external influence on the position of the LCS is possible by alignment changes in the external cavity. In particular, a tilt of the VBG induces a tilt of the wavefront of the returning beam, which should lead to continuous drift of the LCS in a homogeneous system. For a LCS in a trap, the tilt shifts the position where the potential minimum of the combined perturbation (frozen disorder plus tilt) lies and hence the LCS shifts to a new equilibrium position (see [49] for images). As the quantitative analysis in Fig. 6a shows, this shift is quite small. It should be noted also that the tilt is minute, about $0.15 \mathrm{mrad}$ total, leading to a change of ray angle at the VCSEL smaller than $1 \mathrm{mrad}$, much smaller than, e.g., the angular width of a LCS. The shift within the trap is also different for different solitons, which is expected for a disordered system because the curvature of the potential should vary randomly from trap to trap. At some critical tilt angle (larger than typically achievable with the PZT), the LCS disappears. The expectation is that the potential minimum disappears for a critical tilt and the LCS unpins and starts to drift. A corresponding transition between drifting and pinned patterns was found in [96]. In our system, an experimental investigation of the unpinning phenomenon requires simultaneous spatial and temporal resolution and has not been undertaken, yet.

In conclusion, bistable emission spots with high temporal and spatial coherence emerge at the threshold of a VCSEL with frequency-selective feedback. Although their position is affected by the disordered landscape due to the variations of the cavity resonance, these spots maintain rotational symmetry and a common spatial shape and width, i.e. their shape seems to be dominated by the nonlinear process. 
a)

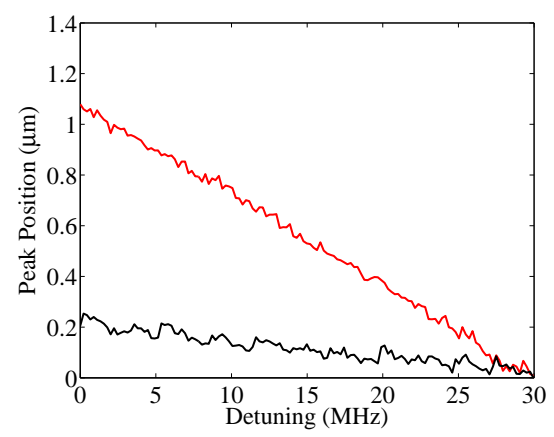

b)

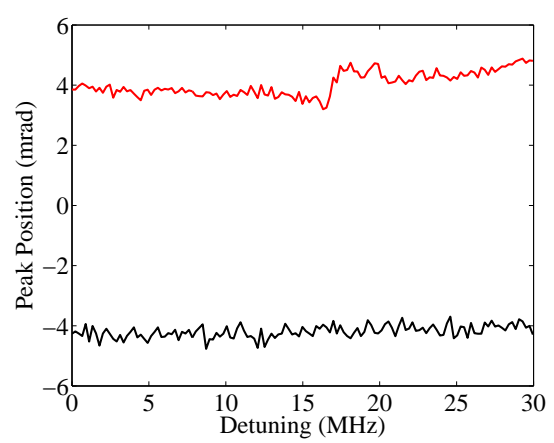

Fig. 6 a) (Color online) Peak position of the near field profile as a function of the tilt angle for LCS 1 (red curve, gray in print) and 2 (black curve); b) Peak position of the far field profile as a function of the tilt angle. The curves are averaged over two runs and most of the undulations are likely to stem from measurement noise, although a deterministic contribution due to small-scale disorder within the trap cannot be ruled out. The zero of the $y$-axis in panel $b$ is set at the center of the two individual angular centres of the far fields of LCS 1 and 2. For a better comparison with the results presented below the horizontal axis is scaled in the change of differential detuning between the two LCS in the external cavity as obtained from Eq. (1). The total tilt is $0.15 \mathrm{mrad}$.

Theoretical results discussed below confirm the existence of self-localized LCS for realistic parameter values. Hence these spots are identified as LCS. The spatial fluctuation of the cavity resonance condition, however, pins LCS to certain locations and leads to a dispersion of thresholds and operating frequencies. Each of these LCS is a coherent emitter but they are mutually incoherent due to the spatial disorder of the background $[49,59,65]$.

\subsection{Phase locking of laser cavity solitons via change of current}

Investigation of LCS interactions were done on pairs of different LCS. We report here results on LCS with a separation distance of 49 (LCS 3 and 4) and $79 \mu \mathrm{m}$ (LCS 1 and 2). The qualitative features of the observations are robust, but the details can vary a fair amount as one would expect since parameters are affected by spatial disorder. We stress that we are only looking at LCS in different traps. The coherence properties of compound, high-order states of LCS in the same trap are a separate issue and more detailed investigation are needed in our system. For the LSA some results are in $[59,86]$.

Two spatially separated LCS are typically incoherent. Then their combined far field is the incoherent sum of the intensity distributions of the two solitons. Under certain conditions (explained in detail below), this changes drastically and a pronounced fringe pattern is visible across the far field intensity profile (Fig. 7c, d). Its wavevector is parallel to the connection line between the two solitons, i.e. the fringes are orthogonal to it. This far-field fringe pattern is stable for longer than 
the exposure time of the CCD (20 ms), typically it can be observed for minutes to hours, once achieved. This evidences phase-coherence between the two LCS over time spans orders of magnitude higher than the intrinsic dynamics (nanoseconds) and also higher than typical time scales of technical noise (microseconds to seconds). A cut through the fringe pattern is taken across the center of the far field intensity. Then a Gaussian profile modulated by a sine-wave is fitted to this cut,

$$
y=y_{0}+A \exp \left[\frac{-\left(\theta-\theta_{0}\right)^{2}}{2 w^{2}}\right]\left[\frac{1+M \sin \left(F\left(\theta-\theta_{0}\right)+P\right)}{1+M}\right] .
$$

Here $y_{0}$ is the offset, $A$ is the amplitude, $x_{0}$ is the peak center, $w$ is the width, $M$ is the modulation depth or fringe visibility, $F$ is the frequency of the sine modulation and $P$ its phase.

Figs. 7a and b show the locking behavior of LCS 1 and 2 during an upward scan of the current. The dominant features of the spectra in Fig. $7 \mathrm{~b}$ are multi-frequency emission and the common red-shift of all modes due to the Ohmic heating discussed earlier. This shift is about $0.44 \mathrm{GHz} / \mathrm{mA}$, about half the value of the free-running laser. This is expected because the grating stabilizes the emission frequency [87, 85]. At low current the LCS emit on two or three different external cavity modes which corresponds to a fringe visibility close to 0.5 . This indicates that the LCS operate on different external cavity modes but share side-bands, i.e. each LCS is not singlemode. Then, as the current is increased, the fringe visibility increases abruptly to reach up to 0.95 . At this point, only one spectral line is strongly dominant and both LCS occupy the same external cavity mode and are then strongly frequency and phase locked. Increasing the current further leads to a jump of one LCS to an adjacent external cavity mode inducing a drop in fringe visibility back to 0.5 . A similar scenario with a transition to full locking occurs at the end and high visibility is reached again. Beyond this point, a third LCS switches on in the detection area thus complicating the interpretation of the results.

From the Fourier theorems, a field distribution shifted by a distance $a$ in the near field acquires a phase-shift of $\exp \left(i k_{\perp} a\right)$ in far field. Hence $a$, the separation of the interference source (here the LCS) in near field and the fringe period $\Delta \theta=2 \pi / F$ are related by

$$
a=\frac{\lambda}{\Delta \theta}
$$

For a far field fringe spacing of $12.6 \mathrm{mrad}$ obtained from the fit one obtains a near field separation of $77.8 \mu \mathrm{m}$ which agrees very well with the measured LCS separation of $79 \mu \mathrm{m}$ thus confirming that the interference comes from the two interacting LCS.

From Fig. 7a it is apparent that the fringe phase fluctuates around a value smaller than $\pi$. As we will discuss below, a locking phase of $\pi$ is typical for two coupled oscillators without detuning while a non-zero detuning changes the locking phase away from $\pi$. The current induced heating is a global parameter and hence it should not change the detuning condition between the two LCS in the VCSEL cavity, in line with the fact that the locking phase is not varying by much. The operating frequency 
a)

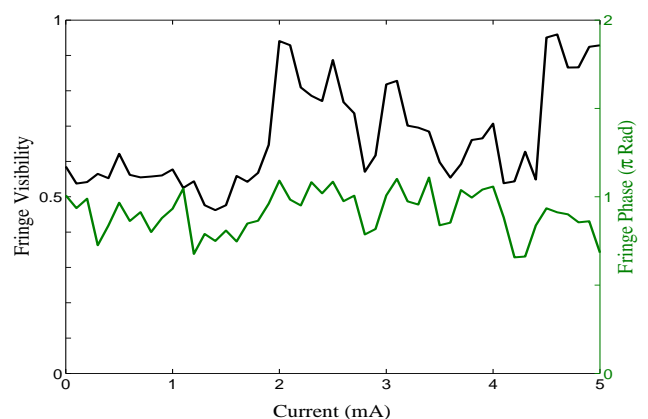

b)

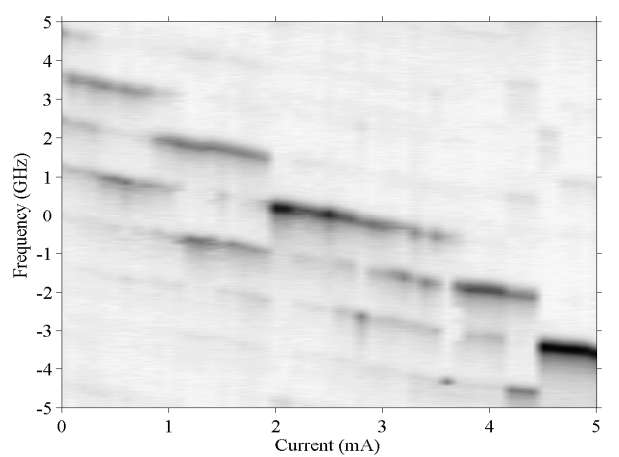

c)

d)

Fig. 7 a) (Color online) Fringe visibility (black) and fringe phase (green curves, gray in print) as a function of current for LCS 1 and 2,79 $\mu \mathrm{m}$ apart. The zero of the current scale corresponds to a current of $380 \mathrm{~mA}$. The fringe visibility and phase are obtained from a fit of far field profiles to Eq. (2). b) Evolution of frequencies; the frequency distribution is obtained from the optical spectra recorded by a Fabry-Perot with a FSR of $10 \mathrm{GHz}$. The frequency separation between side modes corresponds to the free spectral range of the external cavity $(1.23 \mathrm{GHz})$. In these measurements, the Finesse is only 25 due to misalignment (frequency resolution $400 \mathrm{MHz}$ ). c) Far field intensity distribution at $380 \mathrm{~mA}$ corresponding to a fringe visibility of 0.55 . d) Far field intensity distribution at $385.5 \mathrm{~mA}$ corresponding to a fringe visibility of 0.95 . Other parameter: Temperature $69^{\circ} \mathrm{C}$. 
of the solitons, however, is a compromise between the VCSEL cavity resonance and the external cavity resonance leading to a transcendent equation for the operating frequency [97] (see (A.3) of [87] for a VCSEL with FSF). With an initial, position dependent offset, a global shift of the VCSEL resonance conditions can result in a change of relative stability and frequency of the modes of the coupled cavity system (e.g. a destabilization of an external cavity mode for one LCS but not for the other), leading to the possibility of a non-synchronous evolution of soliton frequencies and locking or unlocking. In addition, there might be small local variations either in gain or cavity resonance because the current induced temperature shift is only nominally homogeneous. These considerations indicate that the global parameter 'current' is not a good handle to investigate the locking behavior, but one should look for a 'local knob'.

\subsection{Phase and frequency locking of laser cavity solitons via change of feedback phase}

Since it is experimentally difficult to change the detuning of two LCS by locally changing the properties of the VCSEL itself, the PZT is used to minutely tilt the VBG of the external cavity with respect to the optical axis (Fig. 4). As indicated by Eq. (1) this induces a difference in external cavity length $\Delta L$ for the two solitons therefore leading to a difference of feedback phase and detuning in the external cavity. It is then possible to adjust the frequency difference (i.e. the detuning) between two LCS. Indeed, the locking discussed in the previous section was achieved by aligning the VBG such that a high fringe visibility was obtained for some current ( 2 or $4.5 \mathrm{~mA}$ ). This follows the procedure used to control the detuning of coupled solid-state lasers, see $[4,5]$, but with the additional complication of coupled cavity effects due to the high reflectivity of the VCSEL outcoupling mirror, whereas the gain chip in the solid-state lasers is anti-reflection coated.

As indicated before, the tilts are actually quite small and it turns out that the soliton profiles are essentially unaffected. The width of the near field is constant within $0.3 \mu \mathrm{m}$ (variation $\leq 5 \%$ of a single soliton width) and the width of the far field profiles to within $1.5 \mathrm{mrad}$ ( $6 \%$ of a single soliton width). When tilting the VBG both near and far field profiles of the LCS are not affected while the positions are. As discussed in conjunction with Fig. 6, the positions and beam pointing of the LCS change slightly in the trap, but the changes are small and the differential changes are even smaller. Hence, it seems reasonable to assume that the dominant effect of the tilt is indeed the change of differential feedback phase.

When tilting the VBG a region of phase and frequency locking appears as illustrated in Figs. 8a, c by a range of high fringe visibility in the far field. This region of high fringe visibility can last for seconds to hours depending on parameters. It confirms that locking is a robust feature once achieved by a fine alignment of the VBG. We note that the choice to start the $\mathrm{x}$-axis displaying the detuning parameter with zero is arbitrary. If one considers the locking-dynamics, it would be reasonable 
a)

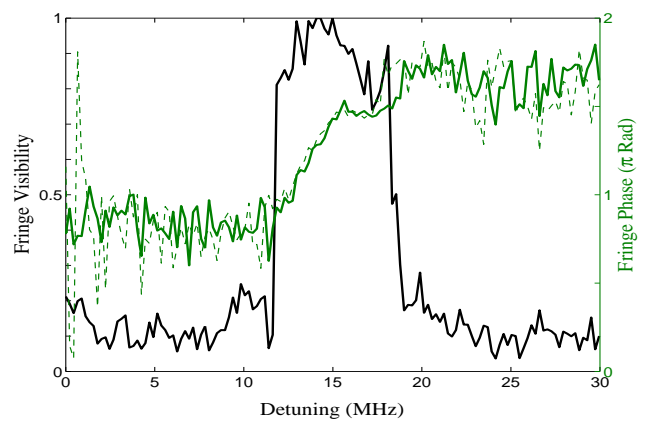

b)

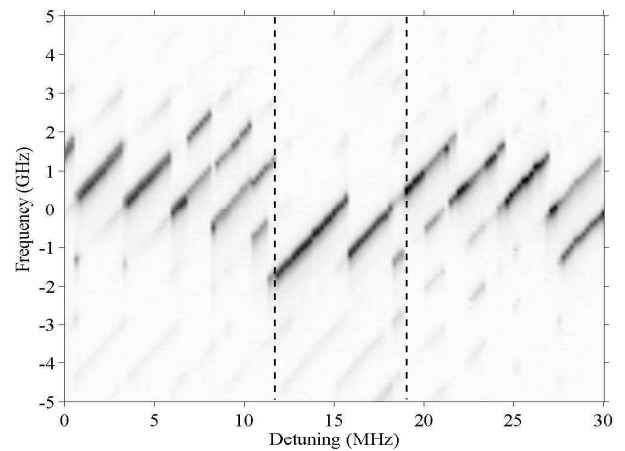

c)

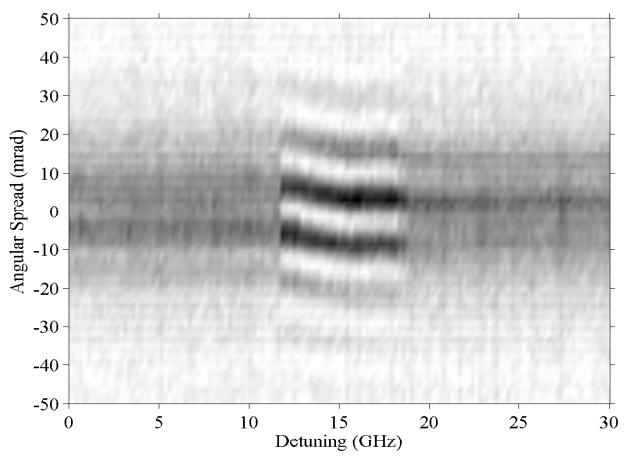

Fig. 8 a) (Color online) Fringe visibility (black) and fringe phase (green curves, gray in print) as a function of the tilt angle that changes the difference between the feedback phases for LCS 1 and 2, $79 \mu \mathrm{m}$ apart. This difference is converted to a frequency scale by multiplying it by the free spectral range of the external cavity thus providing the change of the relative detuning between the two LCS in the external cavity. The zero of this detuning scale is arbitrary. The solid and dashed green curves are obtained for scanning the tilt back and forth. b) Evolution of frequencies, the frequency distribution is obtained from the optical spectra recorded by the FP (Finesse 80). c) Cut through far field intensity distribution orthogonal to fringe orientation. Other parameters: Temperature $69^{\circ} \mathrm{C}$, current $I=373 \mathrm{~mA}$. 
to expect that the zero lies at the center of the locking region. However, as the fringe phase is only approximately symmetric with respect to the center of the locking range and the details of the underlying dynamics are unknown, the position of the zero is somewhat ill-defined and no adjustments were made. Such cautious choice comes at the expense of a slightly awkward labelling: whereas the real detuning decreases in the left half (roughly) of the figure and increases in the right half, the detuning parameter chosen increases all along the $\mathrm{x}$-axis. As qualitatively apparent from Fig. 8c, the fringes shift with detuning within the locking region (within a fringe visibility higher than 0.5 ), i.e. the locking phase changes. The quantitative analysis in Fig. 8a indicates that this variation is nearly linear over most of the range. The width of the locking range is close to $\pi$. If the direction of the scan of the tilt is reversed (see green dashed line in panel a) the locking phase shadows the one obtained in the up-scan, i.e. there is no discernible hysteresis. As we will discuss in the theoretical sections, these features are fingerprints of the Adler locking. The noise of the fringe phase is considerably smaller in the region corresponding to complete locking than in the partially locked regions. For even larger tilts (not achievable with the PZT), the two LCS are completely unlocked and their phases random. Modulations depths of about 5\% are due to noise in the images.

The transitions to and from frequency and phase locking are rather abrupt and one might expect hysteresis at their onset. Due to the mechanical scanning, there is some jitter of the transition point. Hence only one sweep is shown in Fig. 8a and we are currently not in a position to investigate possible hysteresis systematically.

Panel $b$ of Fig. 8 illustrates the evolution of the frequencies of LCS in optical spectra. The first obvious feature is that all spectral lines are shifted due to the tilt of the VBG. The Finesse of the FP is 80, hence the frequency resolution is only about $130 \mathrm{MHz}$. This means that the differential frequency shift of the two LCS cannot be resolved (it is in total about $30 \mathrm{MHz}$ ), but only inferred from its indirect effect via stabilization and destabilization of external cavity modes and the resulting possibility of locking and unlocking. When comparing panel $b$ to panels a and $\mathrm{c}$ one observes that when the fringe visibility is high, the two LCS operate on a single frequency (region within dashed lines in panel b).

There is a jump in the common operation frequency of the LCS by one FSR slightly after $15 \mathrm{GHz}$, where the fringe visibility is nearly maintained. It drops from 0.9-1 to 0.8-0.9. This indicates that the two LCS do a common mode jump retaining phase and frequency locking, though at this point there is a change of behavior in the phase evolution (green solid line in Fig. 8a); it becomes essentially flat.

Outside the locking region the two LCS operate on two different external cavity modes (with a frequency separation of $1.23 \mathrm{GHz}$ between two adjacent modes). In this region the fringe visibility is rather low (below 0.25 ) and the locking is very weak. Some residual coupling via side-modes is probably responsible for this residual partial locking. As indicated, for higher tilts, the visibility drops to a background given by noise on the order of $5 \%$ or less.

Although the transition from locking to unlocking seems to be accompanied by a transition between a one-frequency to a two-frequency spectrum, there are other regions in which the spectrum appears to be predominantly single-humped (possi- 
bly with weak side-modes) but with low fringe visibility. A close inspection however shows that the spectral line is wider there than in the central locking range, by around $45-55 \%$ in the region between $2-6 \mathrm{MHz}$ detuning, and by $27 \%$ in the region at $19.5-20.5 \mathrm{MHz}$ (close to the locking range). This indicates that the two LCS are operating close in frequency so that the difference cannot be resolved within the limited resolution of the FP (about $130 \mathrm{MHz}$ ). There is a third region around $26 \mathrm{MHz}$, where the spectral line seems to be slightly, but not significantly broader, and we conjecture that the frequency difference is below the resolution there.

The basic features of the scenario described for LCS 1 and 2 (79 $\mu \mathrm{m}$ apart) are also typical for other distances and other pairs of LCS. Fig. 9 shows phase and frequency locking for LCS 3 and 4, which are $49 \mu \mathrm{m}$ apart. In this case we observe a far field fringe spacing of $19.1 \mathrm{mrad}$ which corresponds to a near field separation of $51.3 \mu \mathrm{m}$. It again agrees well with the measured LCS separation of $49 \mu \mathrm{m}$. Again, there is a region of nearly complete locking with a high fringe visibility. The phase (within the locking region) is centred around $\pi$ and varies linearly with the detuning from $0.6 \pi$ to $1.4 \pi$ (green curve in panel a, see also panel c). The locking-unlocking transition is accompanied by a transition between a single-frequency and a twofrequency regime. Around a detuning of $4 \mathrm{MHz}$, the transition leads to a single locked state with a visibility of 0.7 dominated by a single mode, although weak side-modes are still present in the spectrum, the latter disappearing with a further increase of the visibility to the 0.9 level. As in Fig. 8b, the LCS perform a common mode-hop within the locking region (around 7.5 MHz detuning), but remain locked. In contrast to the previous case, the phase evolution is not perturbed, i.e. the phase continues to grow approximately linearly. This might be related to the fact that the visibility remains above the 0.9 level, i.e. remains higher than in Fig. 8a.

Before the main locking region, there are already small regions (around $1.5 \mathrm{GHz}$ and $3 \mathrm{GHz}$ ) in which partial locking takes place. An extrapolation of the phase in the main locking region seems to match qualitatively the phase values obtained in these regions. There are also some ranges of non-monotonous behavior of the phase (a decrease with increasing detuning) in the partial locking regions around $3 \mathrm{GHz}$ and $4 \mathrm{GHz}$. The significance of these observations is not clear at the present stage of investigations.

In summary, we have obtained a variety of different dynamical behaviors for LCS pairs with different distances ranging from 30 to $80 \mu \mathrm{m}$. They all share the common feature of a region of nearly complete locking in which the phase is evolving approximately linearly with detuning. The locking is dominantly anti-phase ( $\pi$ locking phase) and the locking phase varies by nearly $\pi$ over the locking range. We will argue in the next sections that these observations are a manifestation of the Adler-scenario. The observed variations in dynamics are expected since important parameters are associated to background disorder that cannot be controlled by the operator. 
a)

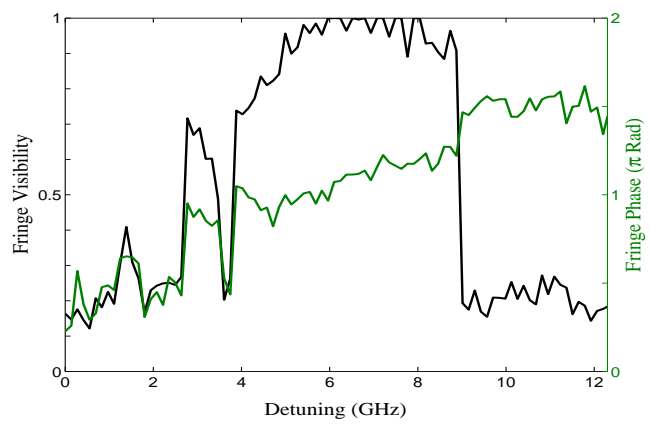

b)

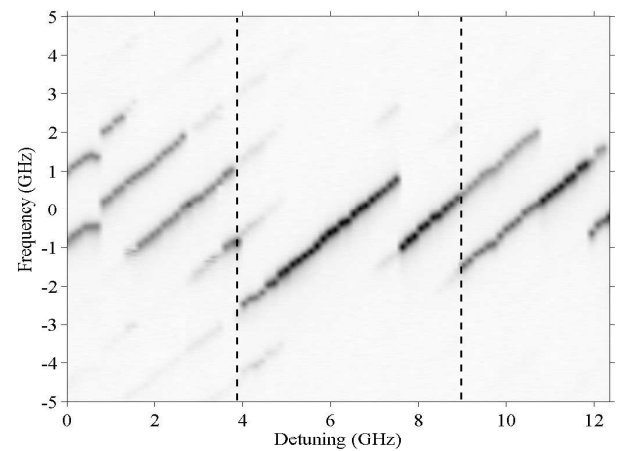

c)

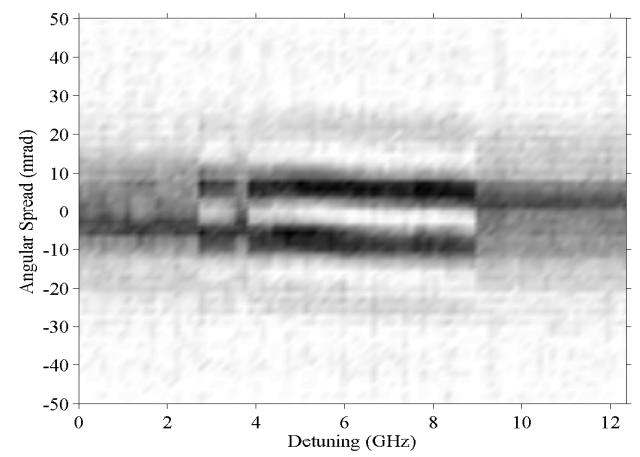

Fig. 9 a) (Color online) Fringe visibility (black) and fringe phase (green curves, gray in print) as a function of the tilt angle that changes the difference between the feedback phases for LCS 3 and 4, $49 \mu \mathrm{m}$ apart. The zero of this detuning scale is arbitrary. The solid and dashed green curves are obtained for scanning the tilt back and forth. b) Evolution of frequencies, the frequency distribution is obtained from the optical spectra recorded by the FP (Finesse 80). c) Cut through far field intensity distribution orthogonal to fringe orientation. Other parameters: Temperature $69^{\circ} \mathrm{C}$, current $I=387 \mathrm{~mA}$. 


\section{Theoretical Description}

\subsection{The semiconductor class-B model}

Since the pioneering work of Lang and Kobayashi [97] a good deal of attention has been paid to the modelling of feedback effects on the dynamics of semiconductor lasers (see for example [98]). Most of the work has been done however by neglecting the spatial degrees of freedom in the transverse direction. Transversespace dependence is critical for LCS and it should be included in any model that describes the set-up discussed in the previous section. Following [52] the dynamical evolution of the intra-VCSEL optical field $E$ and carrier density $N$ of a VCSEL with frequency-selective feedback can be modelled by the following system of coupled partial differential equations and mapping:

$$
\begin{aligned}
\partial_{t} E & =-(1+i \theta) E+i \nabla^{2} E+\sigma(1-i \alpha)(N-1) E+\frac{2 \sqrt{T_{1}}}{\left(T_{1}+T_{2}\right)} F \\
\partial_{t} N & =-\gamma\left[N-J+|E|^{2}(N-1)+D \nabla^{2} N\right] \\
F(t) & =e^{-i \delta \tau_{f}} \hat{G}\left(t-\tau_{f} / 2\right)\left\{-\sqrt{1-T_{1}} F\left(t-\tau_{f}\right)+\sqrt{T_{1}} E\left(t-\tau_{f}\right)\right\}
\end{aligned}
$$

In Eq. (4), $\theta$ is the detuning of the VCSEL cavity with respect to the carrier reference frequency, $\sigma$ is a coupling constant, $\alpha$ is the linewidth enhancement factor, and $T_{1}$ and $T_{2}$ are the transmittivities of the VCSEL mirrors. Note that light propagation in the external cavity is considered here without approximations typical of the LangKobayashi approximations (see also [99]). This allows for the consideration of large feedback reflectivities without incurring in unphysical results (see more details in [52]).

In Eq. (5), $J$ is the injection current normalized to the value at transparency. Time is scaled to the VCSEL cavity lifetime, and $\gamma$ is the ratio of the cavity lifetime to the carrier response time in the VCSEL. The term $D \nabla^{2} N$ describes carrier diffusion but is omitted in what follows. A similar description for field and carriers dynamics was used to model an amplifier CS $[23,100]$. Here, the holding field of the amplifier model is replaced by the feedback field $F$ due to the external Bragg reflector. Space is normalized to the square root of the diffraction parameter. For a low-loss VCSEL, where losses are dominated by the outcoupling, the time scale is of the order of $10 \mathrm{ps}$, while the spatial scale is around $4 \mu \mathrm{m}$ (see also [100]). Since losses due to scattering and background absorption are difficult to quantify we have restricted the analysis to these values of the physical scales.

In Eq. (6), $\delta$ is the external cavity carrier field detuning, $\tau_{f}$ the external roundtrip time (see [52] for a detailed description of the external cavity). The operator $\hat{G}$ describes the frequency-selective operation of the Bragg reflector on the field envelope and is taken to be

$$
\hat{G}(t)\{h(t)\}=\frac{r_{g}}{2 \zeta} \int_{t-2 \zeta}^{t} e^{i \Omega_{g}\left(t^{\prime}-t\right)} h\left(t^{\prime}\right) d t^{\prime},
$$


where $\Omega_{g}$ is the grating central frequency relative to the reference (carrier) frequency (shifted to zero in the following), $\zeta$ the inverse of the filter bandwidth and $r_{g}$ the overall reflection coefficient. Note that this description neglects the transverse wavevector dependence of the reflector response. Transverse effects of free-space propagation (i.e. diffraction) in the external cavity are also disregarded, since in the experimental setup the VCSEL output coupler is imaged directly onto the Bragg reflector as described in the previous section.

Eqs. (4)-(6) have traveling wave solutions as discussed in [52]. It turns out that there are two relevant sets of modes. One set is grouped around the grating frequency and the other around the solitary VCSEL lasing frequency, with a frequency gap in between. The former modes owe their existence to the strong feedback provided by the grating at frequencies close to its central frequency and as a result, have the lowest thresholds. The latter modes exist where the feedback is small and so can be termed VCSEL modes. The frequency gap between grating-determined and VCSEL modes depends on the detuning between VCSEL and grating, as well as on other system parameters.

Under suitable operating conditions it is possible to create a threshold gap between the highest-threshold grating mode and the lowest-threshold VCSEL mode [52]. As a result, a range of currents opens up where the grating-determined modes exist (i.e. the system can lase) but where the laser off state is also stable (i.e. the system can also not lase). In this region of bistability between lasing and non-lasing states it is possible to observe LCS. Numerically one finds that the LCS can be either single- or multi-frequency, depending on system parameters, in 2D as well as 1D [52].

Spatial inhomogeneities, such as those associated to uncontrolled fluctuations in the VCSEL growth process, can be introduced in the model by considering spatial dependence on the model parameters. In particular one can consider in Eq. (4) a spatially dependent detuning, that is $\theta=\theta(x)$ for a $1 \mathrm{D}$ system and $\theta=\theta(x, y)$ for a $2 \mathrm{D}$ one.

\subsection{The simplifed Ginzburg-Landau model}

In this work we are primarily interested in single-frequency laser solutions, in particular solitons. For such solutions the carrier dynamics can be eliminated, reducing the model to a nonlinear equation for $E(x, t)$, coupled to a linear equation for the feedback field $F$. As discussed in [52] and in more detail in [79] these equations have a structure corresponding to the matching of a nonlinear 'soliton' response to a linear 'spectral' response. The latter contains all the gain/loss and phase dependence arising from the delay, grating and mirror properties, while the former describes the effects of current (gain, saturation) and of diffraction. The matching of these responses is associated with the intersection of corresponding curves in a complex plane (C in Fig. 10) describing net gain and phase shift [79]. As illustrated in Fig. 10, the soliton curve is typically rather smooth, originating in a point whose 
location depends smoothly on current. The spectral curve can be highly structured, especially in the case of delayed feedback as is clear when one considers that each external cavity mode must correspond to a different intersection between the soliton line and the spectral curve [52]. Fig. 10 shows an example.

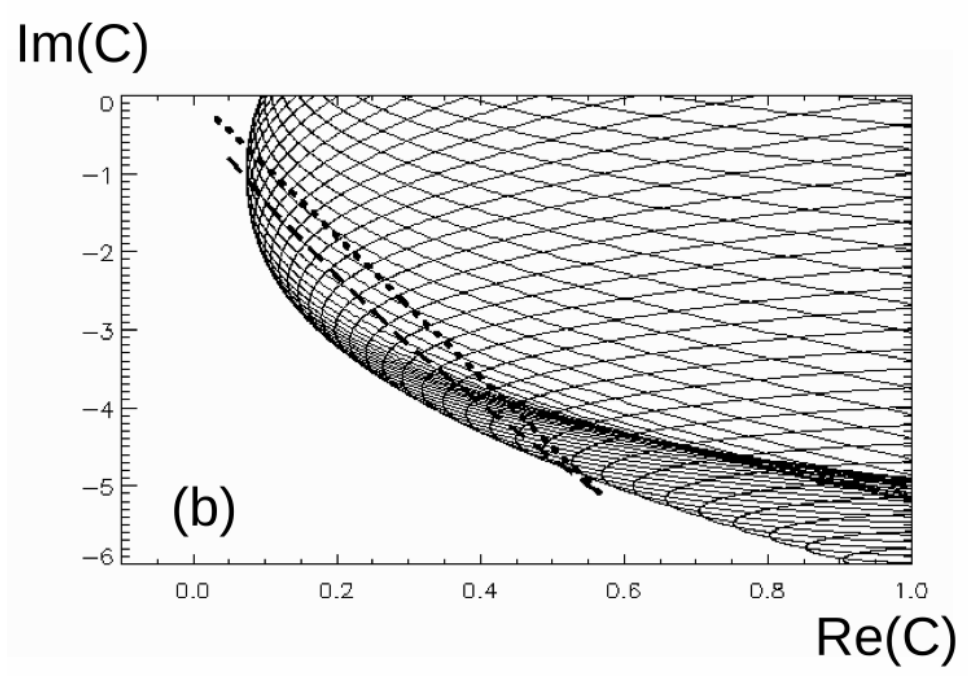

Fig. 10 Spectral curve for the coupled-cavity system showing the soliton line (dashed) and the plane-wave threshold (dotted line). After [52].

It is instructive to investigate the simplest scenario of LCS, which we can obtain by setting aside complications such as delay and high-order nonlinearity. Doing so will also enable us to compare and contrast other models of dissipative soliton interaction, such as those based on generic models such as the cubic-quintic complex Ginzburg-Landau equation (CQGLE) and systems of CGLE [30, 101, 102]. If we eliminate delay and replace the feedback grating with a Lorentzian-response filter, the spectral response curve is broadly similar to the envelope of that in Fig. 10. It is intersected only twice by the soliton line in relevant cases, corresponding to two single-frequency soliton solutions. One (of lower amplitude) is always unstable, while the other may be stable.

A simple model based on this scenario, which captures the basic features of a semiconductor laser with feedback, consists of a cubic complex Ginzburg-Landau equation (CGLE3) linearly coupled to an additional linear filter equation [80, 103]

$$
\begin{aligned}
& \frac{\partial E}{\partial t}=g_{0} E+g_{2}|E|^{2} E+\tilde{D} \frac{\partial^{2}}{\partial x^{2}} E+F+i n(x) E, \\
& \frac{\partial F}{\partial t}=-\lambda F+\tilde{\sigma} E .
\end{aligned}
$$


Here, $E(x, t)$ is the intra-cavity field (we consider only the $1 \mathrm{D}$ case), $g_{0}$ describes linear gain and detuning, $g_{2}$ nonlinear gain saturation and frequency shifts (the ratio $\operatorname{Im}\left(\mathrm{g}_{2}\right) / \operatorname{Re}\left(\mathrm{g}_{2}\right)$ corresponds to the $\alpha$-factor of semiconductor lasers discussed above). The spatial coupling parameter $\tilde{D}$ represents diffusion and/or diffraction depending on its phase: we will set $\tilde{D}=-i$ corresponding to pure diffraction. $F(x, t)$ is the feedback field, $\tilde{\sigma}$ the feedback strength and $\lambda$ the bandwidth of the feedback. Finally, $n(x)$ describes spatial variations of the linear detuning due to local variations in the optical length of the cavity, as discussed in Sect. 2. The time and space coordinates $(t, x)$ are scaled to $1 \mathrm{~ns}$ and $40 \mu \mathrm{m}$, respectively, i.e. the normalized scalings cannot be compared directly between the class-B and the CGLE-F model, but they refer to similar physical scales, after the scalings are undone.

For $n(x)=0$, Eq. (8) has an analytical soliton solution of chirped-sech type [104, 103, 79]:

$$
E=E_{\max }[\cosh (K x)]^{-1-i \beta} e^{i \phi} e^{i \omega t}
$$

where the amplitude $E_{\text {max }}$, the inverse width $K$, the chirp $\beta$ and the frequency $\omega$ are expressed through system parameters. $\phi$ is an arbitrary phase, indicating the phase invariance of the system. The field amplitude decays exponentially in the wings of the soliton (see Fig. 11a below for a particular example). The full width at half maximum of the intensity profile of the soliton is $2 x_{0}$, where $\cosh ^{2}\left(K x_{0}\right)=2$, i.e. $x_{0} \sim 0.88 / K$. The crucial difference between the dissipative soliton (9) and an analogous conservative soliton is the nonzero value of $\beta$ leading to the dependence of the phase on $x$ as illustrated in Fig. 11a. Far from the center, the phase changes linearly with distance and hence repeats with a period of $P=2 \pi /(K \beta)$. Hence only with a moderate to large absolute value of chirp will the soliton phase change significantly within the soliton width $x_{0}$. Recall that an NLSE soliton has flat phase, i.e. zero chirp: broadly speaking, the chirp increases with the ratio of nonlinear gain to nonlinear dispersion.

\section{Theoretical results: Homogeneous Systems}

\subsection{Ginzburg-Landau model}

In this subsection and in subsection 5.1 we consider the pairwise interaction of solitons found in the model (8). We adopt throughout the parameter values of $g_{0}=(-4,28), g_{2}=(-96,-48), \lambda=2.71, \tilde{\sigma}=162.6$ (see [80]). These parameters imply a rather small value (0.5), of the alpha-factor, so chosen in order to enhance the soliton chirp, thus making the interaction stronger and easier to model.

We consider first the ideal case with translational invariance $(n(x)=0)$, where a single soliton has two free parameters, location and phase. We chose a superposition of two solutions of type (9), with the maximum of the left soliton at $x_{1}$, and the maximum of the right soliton at $x_{2}>x_{1}$ as initial conditions for the evolution. We also chose the initial phases $\phi_{1}$ and $\phi_{2}$. The two LCS will mutually interact by 


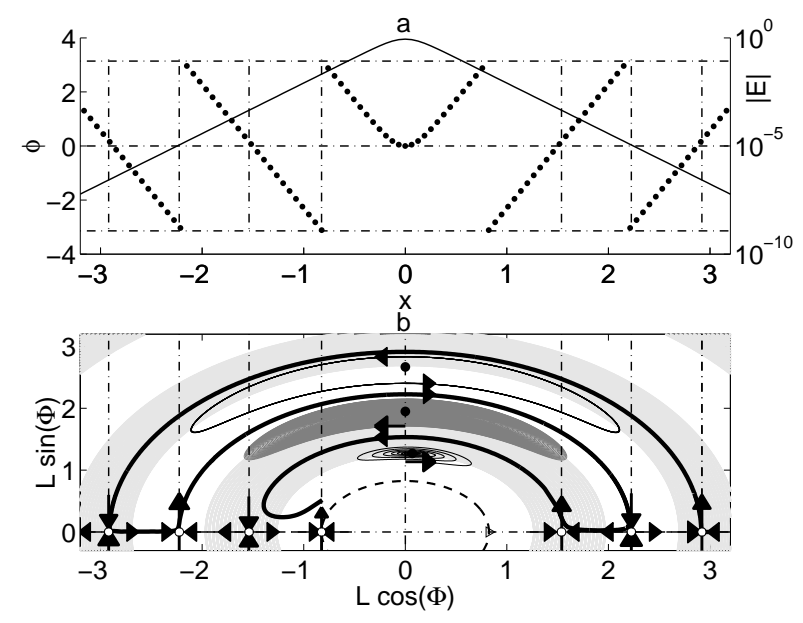

Fig. 11 a) Profile of the soliton field (9) for the parameters listed in the text. Dots show the phase (left vertical axis); solid line shows the amplitude distribution (right vertical axis, semi-logarithmic scale). b) 'interaction plane' of two LCS. The arrows indicate the direction of motion of the system along any trajectory. The dark-grey shaded region is a dense spiral. Black dots near $\Phi=\pi / 2$ are foci, while the while dots (along $\Phi=0$ and $\Phi=\pi$ ) are saddle points. The vertical dash-dotted lines link subplots $a$ and $b$, and confirm that the saddles have the same spatial period as the phase of an isolated soliton. The shading is explained in the text.

providing a perturbation to each other. The interaction can indeed be understood as if each solitons is placed in a potential created by the other, considered as the 'driver'. This means that the field profile of a solitary soliton can be considered as the 'driving potential (and field)' (see Fig. 11a).

The integration of model (8) can be visualized nicely in the 'interaction plane', introduced in [30] and spanned by polar coordinates $L=\left|x_{2}-x_{1}\right|$ and $\Phi=\phi_{2}-\phi_{1}$. The temporal evolution on the interaction plane with different initial values of $L$ and $\Phi$ is presented in Fig. 11b, which depicts key trajectories. It is characterized by a series of foci at $\Phi \approx \pi / 2$ corresponding to moving bound states, and two series of saddles with $\Phi=0$ and $\Phi=\pi$ representing stationary bound states. The stable and unstable manifolds of the saddles coincide with the Goldstone modes of the single soliton. The horizontal manifolds correspond to the soliton translation mode, while the vertical manifolds to that of the local phase. Note that neighboring saddles have opposite stability properties with respect to phase and translation. The light-gray (white) shading in Fig. 11b shows the regions where the active tangential components are directed counterclockwise (clockwise) in the upper half-plane (the sense of rotation is inverted in the lower half-plane that is not shown). It is clear that the location of the saddles is strongly determined by the chirped phase of the individual solitons, which leads to intensity oscillations in their mutual interference profile [29, 34]. We will see how the linewidth enhancement factor $\alpha$ affects the chirped phase and consequently the LCS interaction in subsection 4.2. Note that in 
driven systems without phase symmetry the amplitude already oscillates in the tail of the single soliton as it decays, providing direct means for the formation of bound states at discrete separations $[24,25,27]$.

The interaction phase space diagram of our cubic system with filter looks qualitatively similar to that of the cubic-quintic complex Ginzburg-Landau equation $[34,36]$. We can conclude that this kind of weak-interaction scenario is qualitatively independent from the mechanism of soliton stabilization (quintic nonlinearity or linear filter), although details as soliton width and interaction strength are affected by the details of the nonlinearity quantitatively, of course. This is maybe not surprising since the weak-interaction scenario is dominated by the spatial region in the middle of the solitons where the intensities of both LCS are rather small.

As the principal structure of the interaction plane has been described before [34, 36], we do not go into more detail but mention the deviations of the foci points from $\pm \pi / 2$ (see, in particular, the innermost focus in Fig. 11b). It appears that the foci are slightly shifted from $\phi=\pi / 2$ (asymptotically approaching $\pi / 2$ for $L \rightarrow$ $\infty$ ), while the exact $\pi / 2$ would correspond to fixed points of center type, rather than attracting foci [41]. In summary when the soliton phase is strongly chirped, the dynamics in the interaction plane consists of sets of trajectories which spiral (sometimes very slowly if $L$ is large) into one of a series of foci, each corresponding to a two-soliton bound state which moves transversely. These spirals are bounded by a semi-annulus consisting of a set of four heteroclinic trajectories separate linking adjacent pairs of $(0, \pi)$ saddles. This picture loses validity for very small values of $L$, when the description as a pair of separate, weakly-interacting, solitons becomes problematic and soliton merging takes place [105] (see also the discussion in the following subsection).

For increasing absolute value of the imaginary part of $g_{2}$ (increasing $\alpha$-factor of the semiconductor laser) the phase profile of the solitons becomes flatter and the saddles move to higher distances in the interaction plane. The chosen value of $g_{2}$ here corresponds to $\alpha=0.5$, which is smaller than the experimentally relevant value $(\alpha \approx 2-9$ ) but allows for an effective utilization of the interaction plane.

\subsection{Semiconductor class-B model}

As in the case of the Ginzburg-Landau model described above, the semiconductor class-B model (4)-(6) plus (7) does not contain any optical injection, i.e. it is not an externally driven system. The LCS are then free to choose their own frequency and phase. As such, each LCS can take on a different phase from its neighbouring LCS. We examine here the interaction between two LCS with an initial phase difference at a fixed value while increasing the initial spearation distance. In order to be closer to the experimental realization we use two separate values of the line enhancement factor: $\alpha=5.0$ and $\alpha=9.0$. Other parameter values are: $\zeta=1, \gamma=0.01, T_{1}=$ $0.008, T_{2}=0.0002, \tau_{f}=0.05 n s, r_{g}=0.9$ and $\sigma=0.9$. For $\alpha=9.0 \mathrm{LCS}$ are found for $J=1.63, \theta=1.0$ and $\delta=0.0$ while for $\alpha=5.0 \mathrm{LCS}$ are found for $J=1.61, \theta=$ 
1.0 and $\delta=2.0$. We note that since the reflection of the grating is large $\left(r_{g}=0.9\right)$ and the return times are relatively short, Lang-Kobayashi models cannot be applied here since they would produce spurious solutions with no physical meaning [52]. Model (4)-(7) does not contain Lang-Kobayashi approximations and carefully describes configurations close to the experimental realizations of high feedback reflectivities.

Figure 12 displays the single LCS profiles of amplitude and phase for the two parameter cases corresponding to $\alpha=9.0$ and $\alpha=5.0$. It is clear when compar-
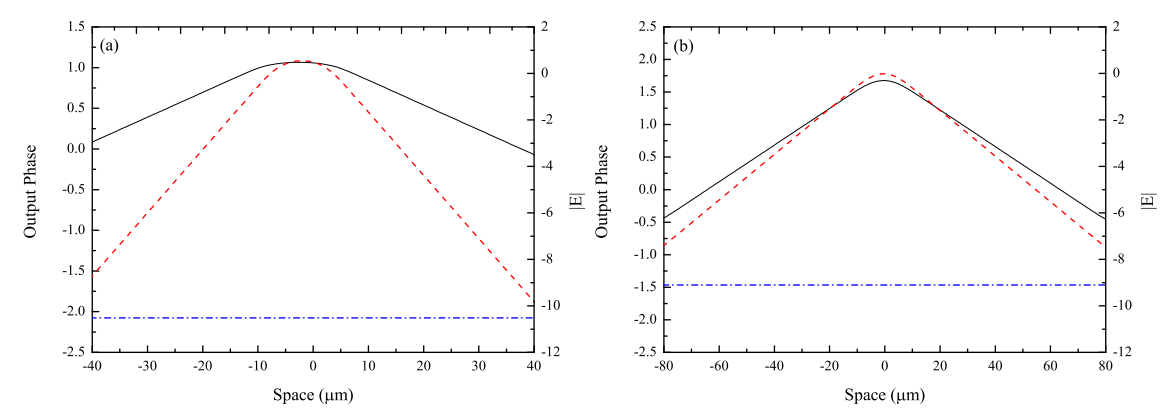

Fig. 12 (Color online) (a) The phase (solid line) and log of the amplitude (dashed line) of a LCS for $\alpha=9.0$. (b) The phase (solid line) and log of the amplitude (dashed line) for a LCS with $\alpha=5.0$. The blue dashed-dotted line corresponds to the noise floor.

ing these figures with Fig. 11a that the LCS phase profiles greatly depend on the linewidth enhancement factor $\alpha$. In the phase profile of the LCS in the GinzburgLandau model with $\alpha=0.5$, the range of phase variation within the width over which the LCS is above the noise floor was larger than $9 \pi$. In the semiconductor class-B models with $\alpha$ factors ten times larger, the total phase variation over which the LCS is above the noise floor is instead just above $\pi$. The much smaller chirp of the LCS has important consequences on the position and dynamical relevance of the saddle points in the interaction $(\operatorname{Lcos}(\Phi), \operatorname{Lsin}(\Phi))$ plane (see Fig. 11b). By linearly fitting the phase profiles of the LCS we have estimated the position of the closest saddle points with growing distances $L$ (taking the results of Fig. 11 as a guidance). In physical units the saddles are expected to be around $L=108 \mu \mathrm{m}$ and $L=117 \mu \mathrm{m}$ for $\alpha=9.0$ and $\alpha=5.0$, respectively, i.e. more than ten times the FWHM size of the LCS.

Numerical simulations of the interaction of two closely placed LCS on a homogeneous background have been performed. Figure 13 shows the temporal evolution of the laser intensity for the process of merging of two LCS when the initial distance of the LCS is $18.8 \mu \mathrm{m}$ for $\alpha=9$. The two peaks oscillate out of phase while approaching each other before merging takes place. Merging of interacting spatial solitons is intrinsically related to their dissipative nature and has been described at length in the case of VCSELs with optical injection in [105]. The phase difference between the LCS first rotates and then becomes ill-defined when one of the two LCS disappears. 

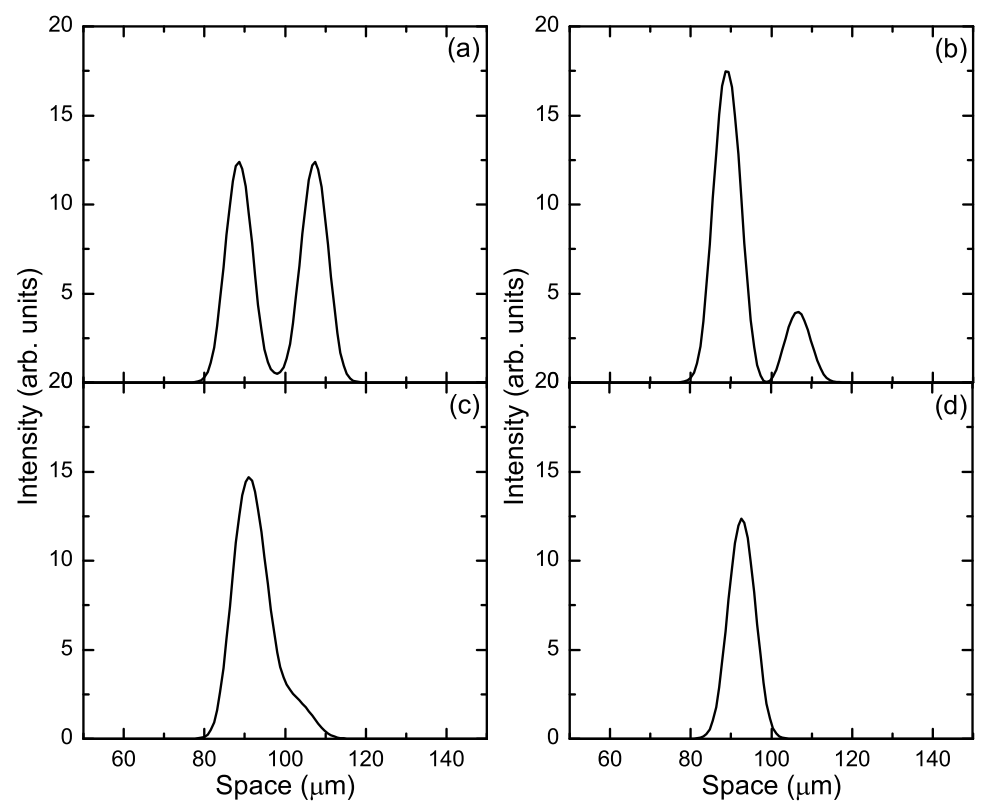

Fig. 13 Time evolution of the intensity of two interacting LCS for an initial distance of $18.8 \mu \mathrm{m}$ and $\alpha=9$ for the class-B VCSEL model with FSF. (a) $t=0 \mathrm{~ns}$, (b) $t=1.2 \mathrm{ns,} \mathrm{(c)} t=1.8 \mathrm{~ns}$ and (d) $t=6 \mathrm{~ns}$. Parameter values are specified in the text.

We have then increased the initial separation distances $L$ of the LCS to $27 \mu \mathrm{m}$ for $\alpha=9.0$ and to $50 \mu \mathrm{m}$ for $\alpha=5.0$. These distances are above the critical values below which LCS merging is observed. Figure 14 shows the time evolution on the interaction plane $(L \cos (\Phi), L \sin (\Phi))$ of simulations starting at $\Phi= \pm 0.1 \pi$.
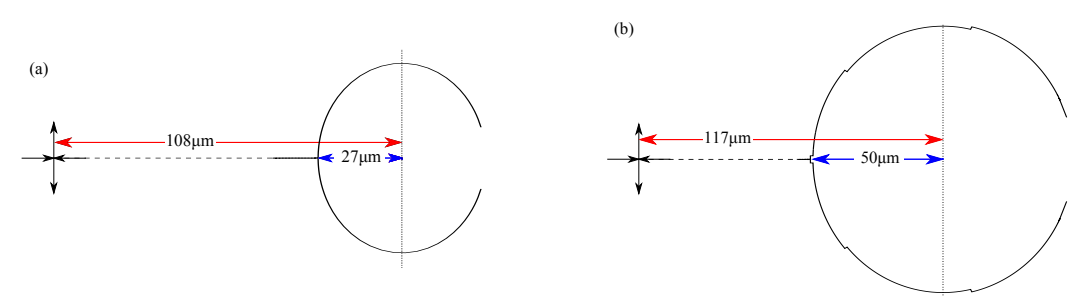

Fig. 14 The interaction plane $(L \cos (\Phi), L \sin (\Phi))$ showing a numerically calculated trajectory and the estimated location of the anticipated closest saddle for (a) $\alpha=9.0$ and (b) $\alpha=5.0$. The origin of the plane is in the center of the circle.

One first observes a quick resetting of the LCS phase difference to the value of $\Phi=\pi$ followed by a very slow progressive separation of the two LCS. The tip of the arrow after phase resetting and along the horizontal axis of Fig. 14 corresponds 
to the final LCS distance after long extended simulations (90 $\mu \mathrm{s})$. This means that the dashed line on the horizontal axis corresponds to regions where the LCS are almost incapable to interact. The situation is similar to what has been observed in numerical simulations for LCS in semiconductor lasers with saturable absorbers and large initial separation distances between the solitons [42]. In our case the LCS reach a distance larger than five soliton diameters at which they do not feel each other any longer. More importantly, the slowly decaying phase profile implies that interacting LCS at physical values of the $\alpha$ factor cannot reach the saddle points that re-direct the phase difference evolution towards the foci at $\Phi=\pi / 2$ in the Ginzburg-Landau model of subsection 4.1.

One cannot refer to the two LCS at the end of the simulations of the semiconductor class-B model of Fig. 14 as 'locked' although the phase difference $\Phi$ is $\pi$, since the LCS are not interacting any longer. Moreover, defects in the growth of the semiconductor material pin LCS to given spatial positions. For these reasons we decided that is was important to study locking of LCS when pinned in material defects as described in details in section 5.

\section{Theoretical results: Inhomogeneous systems and Adler-locking}

\subsection{Ginzburg-Landau model}

We turn now to address the case of practical importance in which the interaction is between solitons trapped by defects. We break the translational invariance by imposing a pinning potential, $n(x)$, which is localized, being zero everywhere except in the intervals $x_{j}-\frac{W}{2}<x<x_{j}+\frac{W}{2}$ with $j=1,2$, where it is given by

$$
n(x)=\frac{n_{j}}{2}\left[\sin \left(-\frac{\pi}{2}+\frac{2 \pi\left(x-x_{j}\right)}{W}\right)-1\right] .
$$

This potential is a smooth function, as shown for example in Figure 16 by a gray line. The width $W$ of each trap is chosen close to the width at half maximum of the LCS. The trap separation $L_{t}=\left|x_{2}-x_{1}\right|$ is obviously an important parameter. We consider the practically-relevant initial condition where there is one soliton in each trap. In terms of the unperturbed $(L, \Phi)$ phase space diagram we are interested in trajectories emanating from $\left(L_{t}, \Phi_{0}\right)$, where $\Phi_{0}$ is arbitrary.

We consider first the symmetric case of two identical traps with $n_{1}=n_{2}=n_{0}$, and examine the changes in the interaction plane trajectories as $n_{0}$ is increased. Since translation is a neutral mode of the unperturbed system, any attractive potential is able to trap an isolated LCS. Two solitons in identical, weak, traps will still interact, and the strength and sign of their interaction depend strongly on the separation $L_{t}$ of their respective traps. As mentioned above, almost all trajectories in the trap-free case are attracted to one of the the foci in Fig. $11 \mathrm{~b}$ for the considered values of $g_{2}$, in accordance with the literature [30,31,34]. The corresponding bound 
states move with a finite speed, and so these states are not destroyed by infinitesimal perturbations, but only by those of finite amplitude. Since almost all trajectories in the interaction plane are attracted to a focus for $n_{0}=0$, they will still do so in the presence of infinitesimal perturbations. Trajectories starting close enough to a $L$ unstable saddle will however be modified by infinitesimal perturbations because the unstable eigenvalue becomes vanishingly small close enough to such a saddle. We can therefore expect that such saddles become stable nodes when $L_{t}$ is close enough to the saddle separation. Such a node corresponds to the two solitons being phaselocked, either in-phase or out-of-phase, depending on $L_{t}$. The basin of attraction of each new node will be infinitesimal for an infinitesimal perturbation, but grows with $n_{0}$. Note that the $L$-stable saddles of the homogeneous system are all $\Phi$-unstable, and will remain so under perturbation.

For finite $n_{0}$, the solitons will phase-lock over a finite range of $L_{t}$ around the saddle-separation. These trap-induced nodes will become the dominant attractors once $n_{0}$ is large enough to destroy the $\pi / 2$ foci thus implying that the solitons remain locked for all times. For strongly-trapped solitons the interaction plane dynamics collapses onto the circle $L=L_{t}$, and the phase difference $\Phi$ is attracted to either 0 or $\pi$, depending on $L_{t}$ but independent of $\Phi_{0}$.

Due to the decrease of interaction strength with distance $L$, the outermost foci and saddles are affected first. For $n_{0}=3.0$ (still an order of magnitude smaller than $\left.\operatorname{Im}\left(g_{0}\right)=28\right)$, the depth of the trap is large enough to prevent drift and to keep the solitons at fixed positions for $L_{t}>1.25$, i.e. outside the central white region in Fig. 11b. At the same time, the perturbation by $n(x)$ is still small enough that the shape of the solitons deviates only slightly from (9) within the trap. Within each of the colored bands (light-gray or white) in Fig. 11b the locking phase is that of the saddle lying in that band, which is phase-stable in the unperturbed problem. This is illustrated in Fig. 15, where the anti-phase state is selected for $L_{t}=1.9$ but the in-phase one for $L_{t}=2.1$.

Since the trapped soliton pairs have a pure phase dynamics, we can attempt to describe the system by an Adler equation [66], the archetypical equation describing synchronization between coupled oscillators. For our case it can be written in the form

$$
\frac{d \Phi}{d t}=\Delta \omega-\varepsilon \sin (\Phi),
$$

where $\Delta \omega$ is the trap-detuning (which is zero for $n_{1}=n_{2}$ ), and $\varepsilon$ is a coupling constant. In-phase and anti-phase solutions are selected for $\Delta \omega=0$, depending on the sign of the coupling. For positive $\varepsilon$ the stable final state is $\Phi=0$, for negative $\varepsilon$ it is $\Phi=\pi$. It is obvious from the above results that the distance between traps affects both the magnitude and sign of the coupling in our system. Corresponding terms appear in the perturbation analysis of the cubic-quintic Ginzburg Landau equation [34]. The survival of the (slightly modified) $\pi / 2$-states for weak perturbations, followed by a transition to in- or anti-phase locking was also observed numerically for the CQCGLE with regular modulations [76, 78].

In- and anti-phase synchronization are the only possibilities in the Adler equation for $\Delta \omega=0$, corresponding to the ideal situation of identical traps with $n_{1}=n_{2}$. 


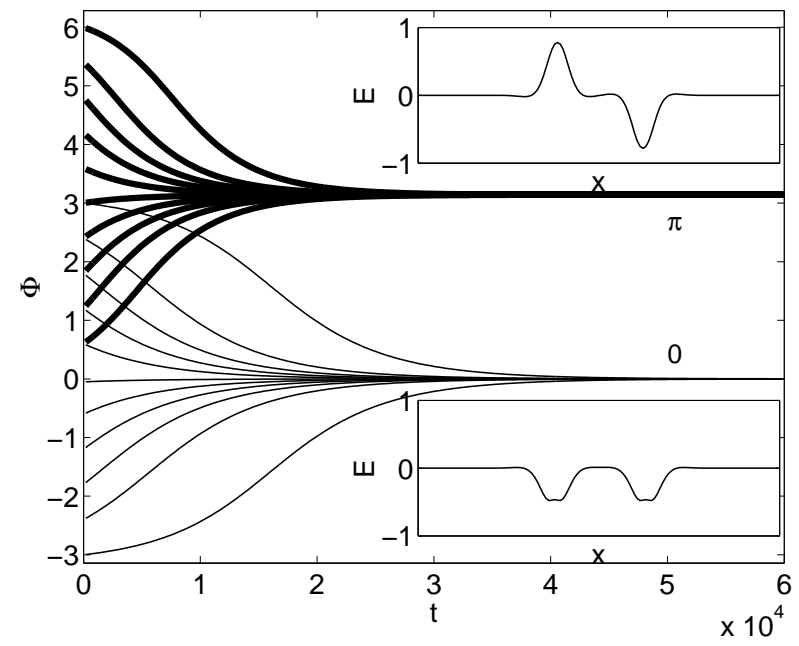

Fig. 15 Dynamics of the phase difference $\Phi$ for different initial values for $L_{t}=1.9$ (upper, bold lines) and for $L_{t}=2.1$ (lower, thin lines). The insets show the instantaneous real part distribution of the corresponding final states.

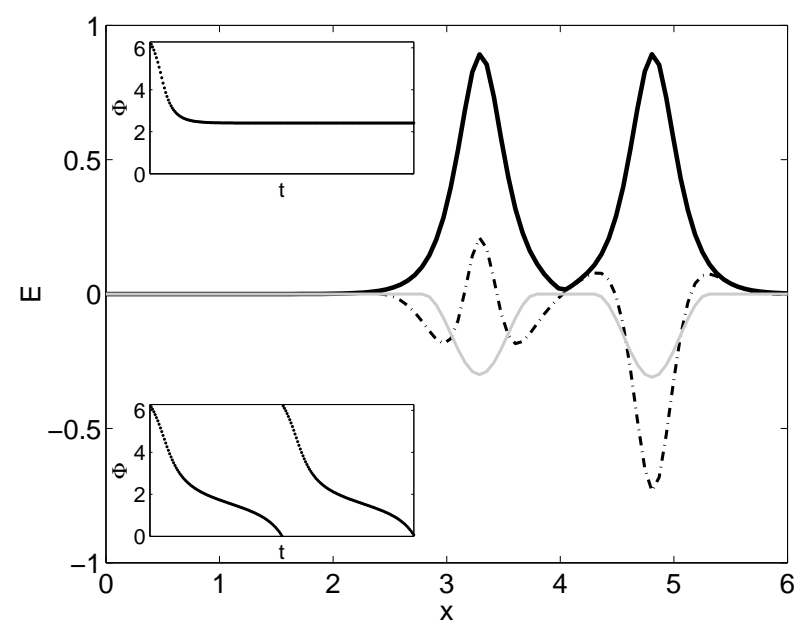

Fig. 16 The bold line is the spatial profile of amplitude of two coupled and synchronized solitons, the dash dotted line is the corresponding instantaneous real part profile. The grey line is the profile of the trapping potential with $n_{1}=3.0$ and $n_{2}=3.1(n(x) / 10$ is plotted). The insets show the dynamics of $\Phi$. The top one is for $L_{t}=1.581$, and displays synchronization. The bottom one is for $L_{t}=1.644-$ the LCS do not synchronize. 
The pinning potential of real systems is however the result of the growth process of the semiconductor material and the traps are not identical. Then we model this fact by introducing a difference in the trap depths (gray line in Fig. 16). This difference leads to the crucial consequence that the natural frequencies of the two LCS are unequal, and so $\Delta \omega$ is finite in the Adler equation. For states with negligible interaction (large $L_{t}$ ), the phase difference evolves as a linear function of time, $\Phi=\Delta \omega t$. Decreasing $L_{t}$ the coupling increases and the dynamics of $\Phi$ starts to deviate from a linear change (see Fig. 16 bottom inset). For small enough $L_{t}$, there is frequency and phase locking (see Fig. 16 top inset). One can see that the system is synchronized now to a phase-locked state (Fig. 16 main panel) with a locking phase which is neither 0 or $\pi$.

For a single soliton in a shallow trap direct numerical integration demonstrates that its frequency depends linearly on the depth of the trap. Hence, for each value of $n_{1}$ and $n_{2}$, we can calculate the frequencies $\omega_{1}$ and $\omega_{2}$ which the LCS would have in the absence of neighbors. We have studied the synchronization dynamics for different detunings $\Delta \omega$ for various choices $n_{2} \neq n_{1}$, and found very good agreement with that predicted by the Adler equation (11) (see section 5.2, Fig. 18, for a plot). The stable steady state of the system (11) gives the simple expression $\sin ^{-1}(\Delta \omega / \varepsilon)$ for the locked value of $\Phi$. Locking obviously becomes impossible for $|\Delta \omega|>|\varepsilon|$, and we can use this limit to compute the coupling constant $\varepsilon$ as a function of trap separation $L_{t}$. We find that the interaction strength has an oscillatory component superimposed on the expected decay with increasing trap separation, which we can attribute to the saddle distribution in the unperturbed problem, see Fig. 11. This is illustrated in Fig. 17.

One can interpret the findings in Figs. 11 and 17 in the way that the coupling coefficient $\varepsilon$ has an (exponentially) damped oscillatory behavior with inter-soliton distance $L_{t}$. In particular this implies that the coupling becomes zero at some distances (at the boundaries between the ring-shaped regions in Fig. 11, where the direction of phase flow in the phase direction changes), at least in leading orders, and the tendency to locking is very weak. It also explains, at least qualitatively, why the points of strongest locking are shifted away from the center of the ring-shaped regions in Fig. 11: The coupling strength is defined by the maximum of the product of the exponential decay and the oscillatory component and hence occurs at smaller distances than the maximum of the oscillation, which determines the ring structures. The decay of coupling strength is related to the diminishing overlap between the soliton tails with increasing distance, the oscillatory component to the change of interference conditions due to chirp. One can also reinterpret the sequence of phase-stable and phase-unstable saddles at $\Phi=0, \pi$ in Fig. 11 as being due to an Adler-type phase selection for zero detuning and an oscillatory sign of the coupling coefficient $\varepsilon$. Indeed, for the LSA and the CQGLE Adler-type equations for the relative phase with an interaction coefficient showing an exponentially damped oscillatory behavior with distance were derived in [41, 34], but with additional terms due to the translational Goldstone mode. Of course, the damped oscillatory behavior of the phase-coupling coefficient with inter-soliton distance in the Adler equation is related to a corresponding behavior of the overall interaction strength between soli- 


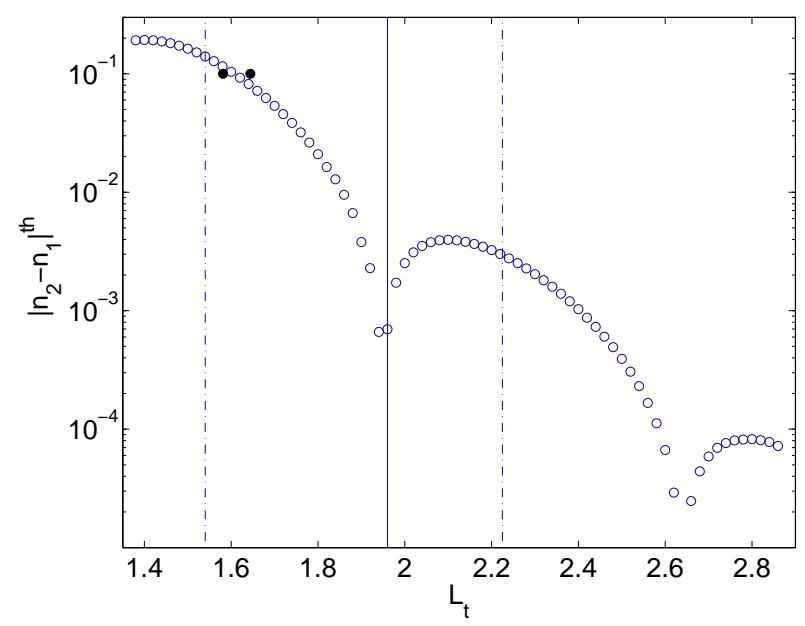

Fig. 17 Critical difference in trap depths for unlocking of LCS vs. distance $L_{t}$ between traps (open circles). The two filled circles correspond to the data of Fig. 16, which straddle the critical curve. The vertical line at $L_{t} \approx 2$ corresponds to the LCS separation in one of the focus states in the unperturbed problem, which appears linked to a local minimum of the locking range. The two dash-dot vertical lines correspond to the adjacent saddles.

tons with inter-soliton distance as described first in [29] and then explored in many of the references given in the introduction.

Taking into account the $L_{t}$ dependence by scaling $\Delta \omega$ by $\varepsilon$, the Adler equation predicts a universal arcsin dependence of the locking phase on the trap-detuning. We find just such a dependence for a broad range of parameters. An example is shown in Fig. 18 for $L_{t}=1.5$, where analogous data for the semiconductor class-B model shows exactly the same Adler signature as discussed in the following section.

Before ending this section, we mention that two LCS in two traps of different depths (or widths) are obviously not entirely equivalent but for the parameters considered the differences are negligible as shown by the profiles in Fig. 16. The same holds for the class-B case discussed below. In the experiment there are actually noticeable variations in width and amplitude between different LCS, although still small, as evidenced in Fig. 5 (see also the text in Sect. 2.2 and [49]).

\subsection{Semiconductor class-B model}

The locking of LCS pinned to background defects as observed experimentally in the VCSEL with frequency-selective feedback in Section 2 and in the simulations of the Ginzburg-Landau model in Section 5.1 is a universal phenomenon and has been found in the semiconductor class-B model of Eq. (4)-(7) too. In order to simulate the presence of the pinning defects, we have modified the field equation to 


$$
\partial_{t} E=-(1+i \theta) E+i \frac{\partial^{2}}{\partial x^{2}} E+\sigma(1-i \alpha)(N-1) E+i n(x) E+\frac{2 \sqrt{T_{1}}}{\left(T_{1}+T_{2}\right)} F,
$$

where the feedback field is still given by Eq. (6) and the trapping potential $n(x)$ is equal to zero everywhere except in the two intervals $x_{j}-W<x<x_{j}+W$ where $n(x)=n_{j}$ with $j=1,2$. The homogeneous case is recovered when $n_{1}=n_{2}=0$. The pinning potential is now a step function of $x$ and the width $2 W$ of the defects is chosen to be around twice the width at half maximum of the LCS. As in the Ginzburg-Landau case, differences between the defects are described by the depths $n_{1}$ and $n_{2}$ of the pinning potential. For $n_{j}$ different from zero, the presence of the pinning potential breaks the translational invariance of each LCS. Small variations of $n_{j}$ from zero lead to small changes in the soliton frequency $\omega$ without modifying its stability properties.

If two trapped defects are close enough in space, the LCS interaction locks their phase difference to values that depend on $\Delta n=n_{1}-n_{2}$ until the potential depth difference is too large to maintain strong interaction. When compared to the case without defects (see Section 4.2) the evolution of the phase difference $\Phi$ between the trapped LCS contains now only a fast relaxation to well determined stationary values that depend on $\Delta n$ and consequently on the frequency difference between the two LCS. The triangles in Fig. 18 show the stationary phase difference $\Phi$ from numerical simulations of Eq. (12) and (5)-(7) when changing the depth of one of the trap while keeping the second one fixed to the value of -0.1 while the distance between the centres of the traps is kept fixed at $\left|x_{2}-x_{1}\right|=31 \mu \mathrm{m}$. LCS phase locking takes place for $|\Delta n|<\Delta n_{t r}$. This locking phenomenon is universal for LCS pinned by defects and the blue circles in Fig. 18 corresponds to numerical simulations of the Ginzburg-Landau model of Section 5.1 under very different conditions of operation. The solid line in Fig. 18 shows an excellent agreement of the Adler equation (11) predictions with the numerical results of both the Ginzburg-Landau and the semiconductor class-B models for two interacting LCS trapped by defects.

In the locked state, LCS have a fixed phase difference and the same frequency. This is demonstrated in Fig. 19 (a) where the optical spectra of two values of $\Delta n$ inside the locked region are shown and corresponding to $\Delta n / \Delta n_{t h}=0$ and 0.99 , respectively. Within the locked region, the spectra of the two LCS overlap exactly indicating a strong interaction. There is a progressive shift of the locked frequency while scanning the Adler region. This matches qualitatively the experimental results in Figs. 8, 9, though the absolute value is much lower. This is not surprising, because the technique used to change detuning in the experiment is different.

Fig. 19 (b) displays the corresponding far field images. Interference fringes between the two locked solitons are clearly visible. A progressive change of the LCS phase difference $\Phi$ (from $\pi$ at $\Delta n / \Delta n_{t h}=0$ to around $1.5 \pi$ at $\Delta n / \Delta n_{t h}=0.99$ ) is reflected in the change in the symmetry of the fringe pattern. All these features of the LCS locked states are confirmed in the Ginzburg-Landau model and, more importantly, in the experiments on VCSELs with frequency-selective feedback presented in Section 2. 


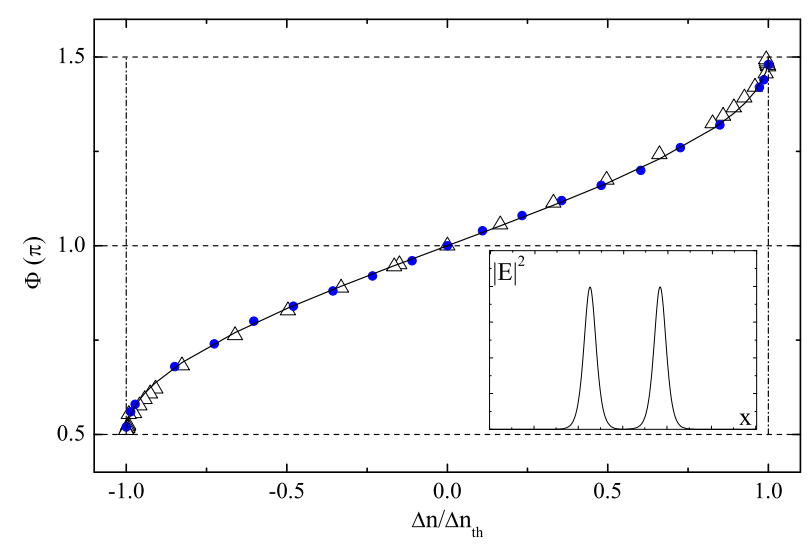

Fig. 18 Locked phase differences $\Phi$ of pinned LCS versus the potential depth difference $\Delta n=$ $n_{1}-n_{2}$ from integration of the semiconductor class-B model (triangles, LCS separation of 4 soliton widths) and Ginzburg-Landau model (blue circles, $\left|x_{2}-x_{1}\right|=1.5$ ). The solid line refers to the Adler equation (11) for negative $\varepsilon$. The inset shows the near-field profile of the $|E|^{2}$ of two interacting LCS. Such profile changes very little across the Adler locking region. From [65].
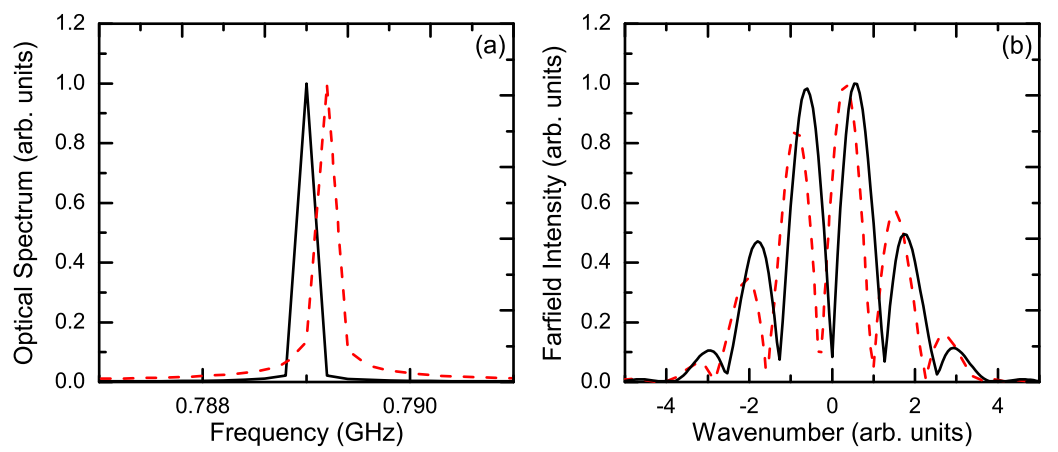

Fig. 19 Optical spectra for a time window of $5 \mu \mathrm{s}$ (a) and far field fringes averaged over $2 \mu \mathrm{s}$ (b), for $\Delta n / \Delta n_{t h}=0$ (solid lines in (a) and (b)) and 0.99 (dashed lines in (a) and (b)). In (a) each line contains the overlap of the spectra of both LCS. Simulations of the semiconductor class-B model.

Both optical spectrum and far-field fringes change greatly as soon as one moves outside of the locking range as shown in Fig. 20 for $\Delta n / \Delta n_{t h}=2$ and 5. The spectra of the two LCS are now clearly separated although a partial overlap of some of the peaks is still present due to the non-uniform evolution of the relative phase. Such feature affects the far-field image, too, where some interference maxima are still visible although progressively disappearing with increasing $\Delta n$. For very large difference of the defect minima, the fringe visibility disappears completely, as expected for two LCS with large frequency separation. 

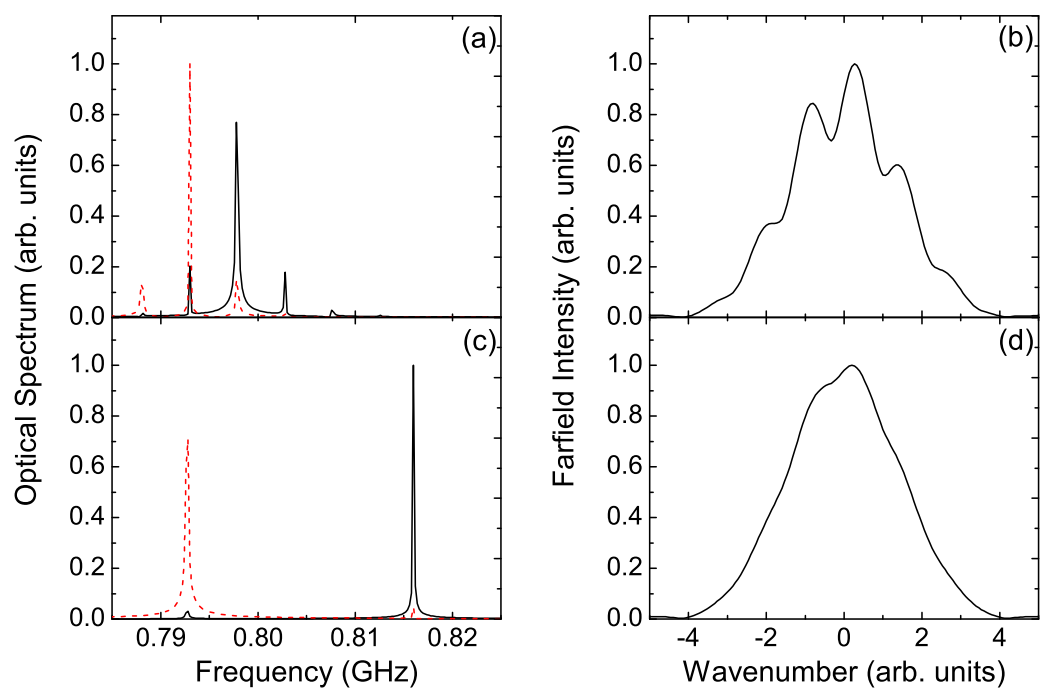

Fig. 20 Optical spectra for a time window of $5 \mu$ s (a)-(c) and far field fringes averaged over $2 \mu \mathrm{s}$ (b)-(d), for $\Delta n / \Delta n_{t h}=2$ ((a)-(b)) and 5 ((c)-(d)). In (a)-(c) the solid and dashed lines correspond to the spectra of each LCS.

The Adler locked state between LCS is a robust feature independent of initial conditions such as initial phases, frequencies and sequential order of creation of the two LCS. Once the locked state is attained, one of the two LCS can be switched off by a short, localized perturbation to the carrier density at its location. Hence, LCS retain their solitonic properties in the phase-locked state in the sense that they are still individually bistable and optically controllable.

\section{Conclusions}

Lasers are oscillators of very high-frequency electromagnetic radiation amplified via stimulated emission in a cavity. Thus, it is not surprising that coupled lasers lock their phases and frequencies in a way similar to oscillators as demonstrated in the seventies through injection locking [9]. Indeed phase and frequency locking of master-slave lasers is almost as old as the laser itself [2]. In the nineties, coupling between adjacent laser elements in arrays also resulted in phase locking typical of coupled oscillators [4].

Here we have demonstrated that locked laser beams can be contained in the same semiconductor laser device in the form of coupled spatial solitons. The specific configuration of choice is that of a VCSEL with frequency-selective feedback provided by a volume Bragg grating. Such systems has been shown to sustain localized structures in the form of bright LCS corresponding to narrow intensity peaks of coherent 
light on a dark background [47, 48, 49]. In principle, well separated LCS are independent micro-laser beams that can be individually addressed and removed thus forming an optical memory. Theoretically, the interaction of two phase-chirped LCS should lead to a phase locked state with a frequency difference close to $\pi / 2$ in a way similar to what is observed in temporal-longitudinal systems [40]. The reality of VCSELs with frequency-selective feedbacks is however quite different from this scenario. First, realistic values of the linewidth enhancement factor $\alpha$ strongly reduce the LCS phase chirp making it impossible to observe the $\pi / 2$ locking even on homogeneous backgrounds. Secondly and more importantly, LCS in real systems are pinned to local defects that are due to the growth process of the semiconductor material. We have shown that the pinning process has two fundamental effects on a single LCS: to break the translational symmetry and to modify its frequency. When these effects are taken into consideration, the picture of the interaction of pinned LCS changes drastically from that of cavity solitons in temporal-longitudinal systems. We demonstrated both experimentally and theoretically that two trapped LCS in VCSELs with frequency-selective feedback display Adler synchronization leading to phase and frequency locking.

We expect similar considerations to apply to solitons in LSA and non-semiconductor systems with phase symmetry such as photorefractive oscillators. Moreover our study uses a continuous model, but synchronization is between discrete entities, the solitons. As such, we have provided a bridge between spatially extended media and coupled, predefined oscillators. Furthermore we note that the LCS are quite peculiar micro-lasers since they are self-localized and bistable so that new interesting dynamics beyond the standard Adler scenario is expected from these properties.

We plan to extend the study of interaction of pinned LCS from two to multiple elements as well as considering their pinning into externally induced regularly and irregularly arranged traps. This can be based on codes already developed for 2D dynamical simulations and stability analysis of single soliton solutions in the CGLE$\mathrm{F}$ as well as class-B models $[52,106,80]$. In view of the random detuning conditions due to the disorder, it can be anticipated that it is impossible, or at least difficult, to achieve locking of more than two LCS by a single, global control parameter (as the VBG tilt). Although we occasionally observed phase-locking of three solitons [107], it would be useful to have control on the local values of the detuning, in addition to the global one used in this work. Previous investigations established that the hysteresis loop of LCS can be shifted to some extent independently by local injection of an external beam [108]. The external beam generates or depletes carriers (depending on wavelength), the refractive index changes and this causes a shift of the cavity resonance (there might be a thermal effect in addition) and finally of switching thresholds. Although not investigated in [108], we expect the change of switching thresholds to be accompanied by a change in frequency. This would open up the intriguing possibility of shaping the disorder dynamically via an external beam structured with a spatial light modulator.

Acknowledgements P.V.P. acknowledges support from SFB 910; C.M., and Y.N. from EPSRC DTA; P.C. from MICINN and Feder (FIS2007-60327, FISICOS, TEC2009-14101, DeCoDicA). 
Y.N. and C.M. were supported by EPSRC DTA/DTG grants. We are grateful to A. G. Vladimirov for useful discussions, R. Jäger (Ulm Photonics) for the devices and N. Radwell for fruitful collaboration in the initial phases of the work. Phase locking of LCS was first described in his thesis [107].

\section{References}

1. H. Haken, Zeitschrift fur Physik 181, 96 (1964)

2. J.W. Crowe, W.E. Ahearn, IEEE J. Quantum Electron. 4, 169 (1968)

3. Z. Jiang, M. McCall, J. Mod. Opt. 39, 159 (1992)

4. L. Fabiny, P. Colet, R. Roy, D. Lenstra, Phys. Rev. A 47, 4287 (1993)

5. M. Möller, B. Forsmann, W. Lange, Chaos, Solitons \& Fractals 10, 825 (1999)

6. A. Pikovsky, M. Rosenblum, J. Kurths, Synchronization - A universal concept in nonlinear sciences, Cambridge Nonlinear Science Series, vol. 12 (Cambridge University Press, Cambridge, 2001)

7. S. Strogatz, I. Steward, Sci. Am. 269, 102 (1993)

8. R.H. Pantell, Proc. IEEE 53, 474 (1965)

9. C.J. Buczek, R.J. Freiberg, M.L. Skolnick, Proc. IEEE 61, 1411 (1973)

10. J.J. Raftery, A.C. Lehman, A.J. Danner, P.O. Leisher, A.V. Giannopoulos, K.D. Choquette, Appl. Phys. Lett. 89, 081119 (2006)

11. L.D.A. Lundeberg, G.P. Lousberg, D.L. Boiko, E. Kapon, Appl. Phys. Lett. 90, 021103 (2007)

12. H. Pier, E. Kapon, Opt. Lett. 22, 546 (1997)

13. K. Otsuka, Y. Miyasaka, T. Narita, S.C. Chu, C.C. Lin, J.Y. Ko, Phys. Rev. Lett. 97, 213901 (2006)

14. P. Colet, R. Roy, Opt. Lett. 19, 2056 (1994)

15. G. VanWiggeren, R. Roy, Science 20, 1198 (1998)

16. J. Goedgebuer, L. Larger, H. Porte, Phys. Rev. Lett. 80, 2249 (1998)

17. A. Argyris, D. Syvridis, L. Larger, V. Annovazzi-Lodi, P. Colet, I. Fischer, J. Garcia-Ojalvo, C.R. Mirasso, L. Pesquera, A. Shore, Nature 437, 343 (2005)

18. G.I. Stegeman, M. Segev, Science 286, 1518 (1999)

19. N. Akhmediev, A. Ankiewicz, Dissipative solitons, Lecture Notes in Physics, vol. 661 (Springer, Berlin, 2005)

20. N. Akhmediev, A. Ankiewicz, Dissipative Solitons: From Optics to Biology and Medicine, Lecture Notes in Physics, vol. 751 (Springer, 2008)

21. N.N. Rosanov, Spatial hysteresis and optical patterns. Springer Series in Synergetics (Springer, Berlin, 2002)

22. K. Staliunas, V. Sánchez-Morcillo, Transverse Patterns, Springer Tracts in Modern Physics, vol. 183 (Springer, 2003)

23. S. Barland, J.R. Tredicce, M. Brambilla, L.A. Lugiato, S. Balle, M. Giudici, T. Maggipinto, L. Spinelli, G. Tissoni, T. Knödel, M. Miller, R. Jäger, Nature 419, 699 (2002)

24. N.N. Rosanov, G.V. Khodova, J. Opt. Soc. Am. B 7, 1057 (1990)

25. B. Schäpers, M. Feldmann, T. Ackemann, W. Lange, Phys. Rev. Lett. 85, 748 (2000)

26. D.Y. Tang, M.W. S., H.Y. Tam, P.D. Drummond, Phys. Rev. A 64, 033814 (2001)

27. P.L. Ramazza, E. Benkler, U. Bortolozzo, S. Boccaletti, S. Ducci, F.T. Arecchi, Phys. Rev. E 65, $066204(2002)$

28. C.P. Schenk, P. Schütz, M. Bode, H.G. Purwins, Phys. Rev. E 57, 6480 (1998)

29. B.A. Malomed, Phys. Rev. A 44, 6954 (1991)

30. N. Akhmediev, A. Ankiewicz, J.M. Soto-Crespo, Phys. Rev. Lett. 79, 4047 (1997)

31. V.V. Afanasjev, B.A. Malomed, P.L. Chu, Phys. Rev. E 56, 6020 (1997)

32. N. Akhmediev, F. Zen, P. Chu, Opt. Commun. 201, 217 (2002) 
33. H. Leblond, A. Komarov, M. Salhi, A. Haboucha, F. Sanchez, J. Opt. A 8, 319326 (2006)

34. D. Turaev, A.G. Vladimirov, S. Zelik, Phys. Rev. E 75, 045601(R) (2007)

35. J.M. Soto-Crespo, P. Grelu, N. Akhmediev, N. Devine, Phys. Rev. E 75, 016613 (2007)

36. A. Zavyalov, R. Iliew, O. Egorov, F. Lederer, Phys. Rev. A 79, 053841 (2009)

37. P. Grelu, F. Belhache, F. Gutty, J.M. Soto-Crespo, Opt. Lett. 27, 966 (2002)

38. J.M. Soto-Crespo, N. Akhmediev, P. Grelu, F. Belhache, Opt. Lett. 28, 1757 (2003)

39. D.Y. Tang, B. Zhao, L.M. Zhao, H.Y. Tam, Phys. Rev. A 64, 016616 (2005)

40. P. Grelu, N. Akhmediev, Nat. Phot. 6, 84 (2012)

41. A.G. Vladimirov, G.V. Khodova, N.N. Rosanov, Phys. Rev. E 63, 056607 (2001)

42. H. Vahed, R. Kheradmand, H. Tajalli, G. Tissoni, L.A. Lugiato, F. Prati, Phys. Rev. A 84, 063814 (2011)

43. V.Y. Bazhenov, V.B. Taranenko, M.V. Vasnetsov, Proc. SPIE 1806, 14 (1992)

44. M. Saffman, D. Montgomery, D.Z. Anderson, Opt. Lett. 19, 518 (1994)

45. V.B. Taranenko, K. Staliunas, C.O. Weiss, Phys. Rev. A 56, 1582 (1997)

46. G. Slekys, K. Staliunas, C.O. Weiss, Opt. Commun. 149, 113 (1998)

47. Y. Tanguy, T. Ackemann, W.J. Firth, R. Jäger, Phys. Rev. Lett. 100, 013907 (2008)

48. Y. Tanguy, N. Radwell, T. Ackemann, R. Jäger, Phys. Rev. A 78, 023810 (2008)

49. N. Radwell, T. Ackemann, IEEE J. Quantum Electron. 45, 1388 (2009)

50. P.V. Paulau, A.J. Scroggie, A. Naumenko, T. Ackemann, N.A. Loiko, W.J. Firth, Phys. Rev. E 75, 056208 (2007)

51. P.V. Paulau, D. Gomila, T. Ackemann, N.A. Loiko, W.J. Firth, Phys. Rev. E 78, 016212 (2008)

52. A.J. Scroggie, W.J. Firth, G.L. Oppo, Phys. Rev. A 80, 013829 (2009)

53. P. Genevet, S. Barland, M. Giudici, J.R. Tredicce, Phys. Rev. Lett. 101, 123905 (2008)

54. T. Elsass, K. Gauthron, G. Beaudoin, I. Sagnes, R. Kuszelewicz, S. Barbay, Appl. Phys. B 98, $327(2010)$

55. M. Bache, F. Prati, G. Tissoni, R. Kheradmand, L.A. Lugiato, I. Protsenko, M. Brambilla, Appl. Phys. B 81, 913 (2005)

56. L. Columbo, L. Gil, P. Genevet, Eur. Phys. J. D 59, 97107 (2010)

57. T. Ackemann, G.L. Oppo, W.J. Firth, Adv. Atom. Mol. Opt. Phys. 57, 323 (2009)

58. S. Barbay, R. Kuszelewicz, J.R. Tredicce, Adv. Opt. Tech. 2011, 628761 (2011)

59. P. Genevet, M. Turconi, S. Barland, M. Giudici, J.R. Tredicce, Eur. Phys. J. D 59, 109 (2010)

60. N.N. Rosanov, Proc. SPIE 1840, 130 (1991)

61. W.J. Firth, A.J. Scroggie, Phys. Rev. Lett. 76, 1623 (1996)

62. F. Pedaci, S. Barland, E. Caboche, P. Genevet, M. Giudici, J.R. Tredicce, T. Ackemann, A.J. Scroggie, W.J. Firth, G.L. Oppo, G. Tissoni, R. Jäger, Appl. Phys. Lett. 92, 011101 (2008)

63. F. Pedaci, G. Tissoni, S. Barland, M. Giudici, J.R. Tredicce, Appl. Phys. Lett. 93, 111104 (2008)

64. T. Ackemann, N. Radwell, Y. Noblet, R. Jäger, Opt. Lett. 37, 1079 (2012)

65. P.V. Paulau, C. McIntyre, Y. Noblet, N. Radwell, W.J. Firth, P. Colet, T. Ackemann, G.L. Oppo, Phys. Rev. Lett. 108, 213904 (2012)

66. R. Adler, Proc. IRE 34, 351 (1946)

67. P. Coullet, D. Daboussy, J.R. Tredicce, Phys. Rev. E 58, 5347 (1998)

68. D. Goulding, S.P. Hegarty, O. Rasskazov, S. Melnik, M. Hartnett, G. Greene, J.G. McInerney, D. Rachinskii, G. Huyet, Phys. Rev. Lett. 98, 153903 (2007)

69. J. Thévenin, M. Romanelli, M. Vallet, M. Brunel, T. Erneux, Phys. Rev. Lett. 107, 104101 (2011)

70. A. Schwache, F. Mitschke, Phys. Rev. E 55, 7720 (1997)

71. F. Leo, S. Coen, P. Kockaert, S.P. Gorza, P. Emplit, M. Haelterman, Nat. Phot. 4, 471 (2010)

72. W.J. Firth, Nat. Phot. 4, 415 (2010)

73. A. Shipulin, G. Onishchukov, B.A. Malomed, J. Opt. Soc. Am. B 14, 3393 (1197)

74. B.A. Malomed, A. Shipulin, Opt. Commun. 162, 140 (2009)

75. W.W. Hsiang, C.Y. Lin, Y. Lai, Opt. Lett. 31, 1627 (2006)

76. W. Chang, N. Akhmediev, S. Wabnitz, Phys. Rev. A 80, 013815 (2009)

77. W. Chang, N. Akhmediev, S. Wabnitz, M. Taki, J. Opt. Soc. Am. B 26, 2204 (2009) 
78. Y.J. He, B.A. Malomed, D. Mihalache, B. Liu, H.C. Huang, H. Yang, H.Z. Wang, Opt. Lett. 34, 2976 (2009)

79. W.J. Firth, P.V. Paulau, Eur. Phys. J. D 59, 13 (2010)

80. P.V. Paulau, D. Gomila, P. Colet, B.A. Malomed, W.J. Firth, Phys. Rev. E 84, 036213 (2011)

81. M. Grabherr, R. Jäger, M. Miller, C. Thalmaier, J. Herlein, K.J. Ebeling, IEEE Photon. Technol. Lett. 10, 1061 (1998)

82. M. Grabherr, M. Miller, R. Jäger, R. Michalzik, U. Martin, H.J. Unold, K.J. Ebeling, IEEE J. Sel. Top. Quantum Electron. 5(3), 495 (1999)

83. M. Schulz-Ruhtenberg, Y. Tanguy, K.F. Huang, R. Jäger, T. Ackemann, J. Phys. D: Appl Phys. 42, 055101 (2009)

84. T. Ackemann, S. Barland, M. Cara, S. Balle, J.R. Tredicce, R. Jäger, P.M. Grabherr, M. Miller, K.J. Ebeling, J. Opt. B: Quantum Semiclass. Opt. 2, 406 (2000)

85. Y. Noblet, T. Ackemann, Phys. Rev. A 85, 05381 (2012). Accepted for publication.

86. P. Genevet, S. Barland, M. Giudici, J.R. Tredicce, Phys. Rev. Lett. 104, 223902 (2010)

87. A. Naumenko, N.A. Loiko, M. Sondermann, K.F. Jentsch, T. Ackemann, Opt. Commun. 259, 823 (2006)

88. A.V. Naumenko, N.A. Loiko, T. Ackemann, Phys. Rev. A 76, 023802 (2007)

89. C.H. Henry, IEEE J. Quantum Electron. 18, 259 (1982)

90. L.A. Lugiato, Progress in Optics XXI pp. 70-216 (1984)

91. R. Neubecker, T. Tschudi, J. Mod. Opt. 41, 885 (1994)

92. R. Kuszelewicz, I. Ganne, I. Sagnes, G. Slekys, Phys. Rev. Lett. 84, 6006 (2000)

93. D. Sanvitto, D.N. Krizhanovskii, D.M. Whittaker, S. Ceccarelli, M.S. Skolnick, J.S. Roberts, Phys. Rev. B 73, 241308(R) (2006)

94. J.P. Weber, K. Malloy, S. Wang, IEEE Photon. Tech. Lett. 2, 162 (1990)

95. J.L. Oudar, R. Kuszelewicz, B.G. Sfez, J.C. Michel, R. Planel, Opt. Quantum Elect. 24, S193 (1992)

96. J.P. Seipenbusch, T. Ackemann, B. Schäpers, B. Berge, W. Lange, Phys. Rev. A 56, R4401 (1997)

97. R. Lang, K. Kobayashi, IEEE J. Quantum Electron. 16, 347 (1980)

98. D.M. Kane, K.A. Shore, Unlocking Dynamical Diversity: Optical Feedback Effects on Semiconductor Lasers (Wiley, New York, 2005)

99. M. Giudici, S. Balle, T. Ackemann, S. Barland, J.R. Tredicce, J. Opt. Soc. Am. B 16, 2114 (1999)

100. L. Spinelli, G. Tissoni, M. Brambilla, F. Prati, L.A. Lugiato, Phys. Rev. A 58, 2542 (1998)

101. J. Atai, B.A. Malomed, Phys. Rev. E 54, 4371 (1996)

102. H. Sakaguchi, B.A. Malomed, Physica D 154, 229 (2001)

103. J. Atai, B.A. Malomed, Phys. Lett. A 246, 412 (1998)

104. N.R. Pereira, L. Stenflo, Phys. Fluids 20, 1733 (1998)

105. C. McIntyre, A.M. Yao, G.L. Oppo, F. Prati, G. Tissoni, Phys. Rev. A 81, 013838 (2010)

106. P.V. Paulau, D. Gomila, P. Colet, N.A. Loiko, N.N. Rosanov, T. Ackemann, W.J. Firth, Opt. Exp. 18, 8859 (2010)

107. N. Radwell, Characteristics of a cavity soliton laser based on a VCSEL with frequency selective feedback. Ph.D. thesis (2010)

108. N. Radwell, P. Rose, C. Cleff, C. Denz, T. Ackemann, Opt. Exp. 18, 23121 (2010) 